(C) 2018. This manuscript version is made available under the CC-BY-NC-ND 4.0 license http://creativecommons.org/licenses/by-nc-nd/4.0/

\title{
Optimal spectral approximation of $2 n$-order differential operators by mixed isogeometric analysis
}

\author{
Quanling Deng, ${ }^{\mathrm{a}, \mathrm{b}, *}$, Vladimir Puzyrever, ${ }^{\mathrm{a}, \mathrm{b}}$, Victor Calo ${ }^{\mathrm{a}, \mathrm{b}, \mathrm{c}}$ \\ ${ }^{a}$ Applied Geology, Curtin University, Kent Street, Bentley, Perth, WA 6102, Australia \\ ${ }^{b}$ Curtin Institute for Computation, Curtin University, Kent Street, Bentley, Perth, WA 6102, \\ Australia \\ ${ }^{c}$ Mineral Resources, Commonwealth Scientific and Industrial Research Organisation (CSIRO), \\ Kensington, Perth, WA 6152, Australia
}

\begin{abstract}
We approximate the spectra of a class of $2 n$-order differential operators using isogeometric analysis in mixed formulations. This class includes a wide range of differential operators such as those arising in elliptic, biharmonic, Cahn-Hilliard, Swift-Hohenberg, and phasefield crystal equations. The spectra of the differential operators are approximated by solving differential eigenvalue problems in mixed formulations, which require auxiliary parameters. The mixed isogeometric formulation when applying classical quadrature rules leads to an eigenvalue error convergence of order $2 p$ where $p$ is the order of the underlying B-spline space. We improve this order to be $2 p+2$ by applying optimallyblended quadrature rules developed in [1, 2] and this order is an optimum in the view of dispersion error. We also compare these results with the mixed finite elements and show numerically that the mixed isogeometric analysis leads to significantly better spectral approximations.
\end{abstract}

Keywords: Isogeometric analysis, finite elements, differential operators, eigenvalue problem, spectral approximation, quadratures

\section{Introduction}

The finite element method (FEM) is a widely used and highly effective numerical technique for approximate solutions of boundary value problems. The theory of FEM has been extensively developed during the last 60 years. Nowadays, many different variants of FEM are used for the solutions of various complex linear and nonlinear problems. A special group of FEM techniques is the mixed finite element methods [37]. The word "mixed" in this case indicates that there are extra functional spaces used to approximate different solution variables. Traditionally these could be the function and its gradient where each is approximated with a different discrete representation. The literature on

\footnotetext{
* Corresponding author

Email addresses: Quanling.Deng@curtin.edu.au (Quanling Deng), Vladimir.Puzyrev@curtin.edu.au (Vladimir Puzyrev), Victor.Calo@curtin.edu.au (Victor Calo) 
mixed finite element methods is quite vast and ranges from the first studies in the 1970s by Brezzi 3], Babuška [8, Crouzeix and Raviart [9] to recent contributions [7, 10 13. A large amount of research has been devoted to various stabilization techniques for the mixed methods [10, 14, 19] as well as their error estimates 20, 21.

The mixed formulation is naturally used for problems with two independent variables such as velocity and pressure in the Stokes equations. In other popular cases, the second variable is the first or the second derivative of the original variable and approximating it directly in the problem formulation has physical interest. For example, in elasticity, both the stress and the displacement can enter the formulation as primitive variables and they are linked by an $L^{2}$ projection. Another example is the Darcy flow equation where the mixed variational formulation is posed in terms of the function spaces $L^{2}(\Omega) / \mathbb{R}$ and $\mathbf{H}($ div,$\Omega)$ for the pressure and velocity, respectively (though other options are possible as well, see, e.g., [17]). The mixed finite element framework allows to preserve mass conservation, a property that is important in fluid flow problems [17, 22, 23] and makes the mixed methods competitive numerical techniques in many engineering applications. The mixed problem discretization leads to a linear algebraic system of the saddle point form

$$
\left[\begin{array}{cc}
\mathbf{A} & \mathbf{B}^{T} \\
\mathbf{B} & \mathbf{0}
\end{array}\right]\left[\begin{array}{l}
\mathbf{x} \\
\mathbf{y}
\end{array}\right]=\left[\begin{array}{l}
\mathbf{f} \\
\mathbf{g}
\end{array}\right]
$$

The mixed isogeometric analysis is a relatively unexplored topic. Again, most of the work in this area focuses on the fluid flow problems where various isogeometric formulations were applied to the Stokes problem [24 27]. Recent advances include mixed isogeometric formulations for elasticity 28 and poromechanics [29, 30. In this field, coupling the fluid pressure and the solid deformation, mixed isogeometric formulations violate the inf-sup condition and suffer from numerical instabilities in the incompressible and the nearly incompressible limit. To overcome the numerical instabilities, the projection methods 31 or subdivision-stabilized NURBS discretization can be incorporated 29. In 32, the authors use a mixed isogeometric formulation to solve the Cahn-Hilliard equation in their overall Navier-Stokes-Cahn-Hilliard model problem.

The solution of high-order partial differential equations (PDEs) attracted a lot of attention in recent years. An important subclass is the $2 n$-order PDE, which for $n=$ 1, 2, 3 reduces to different classical PDEs including Laplacian, Allen-Cahn, biharmonic, Cahn-Hilliard, Swift-Hohenberg, phase-field crystal and other problems. Previous work of numerical analysis typically focused on each of these problems separately (e.g., 3336]). Only a limited number of investigations exists for a general $2 n$-order problem (e.g., for phase-field models [37]). For example, non-degeneracy and uniqueness of its periodic solutions were studied in 38 .

Dispersion-minimizing methods based on modified integration rules for reducing dispersion error have been developed previously for classical FEM [39, 40 and isogeometric analysis [1, 2. The dispersion error is reduced by blending two standard quadrature rules or using special quadrature rules [41. For the standard finite and isogeometric elements, these optimal dispersion methods lead to two additional orders of error convergence (superconvergence) in the eigenvalues, while the eigenfunction errors do not degenerate.

In this paper, we utilize the mixed FEM framework for a general $2 n$-order linear differential eigenvalue problem. We develop the mixed isogeometric framework for these 


\section{Problem statement}

Let $\Omega \subset \mathbb{R}^{d}, d=1,2,3$, be a bounded domain with Lipschitz boundary $\partial \Omega$. We consider a class of $2 n$-order linear differential eigenvalue problems: Find $\lambda$ and non-zero $u$ satisfying

$$
\mathcal{L} u=\lambda u \quad \text { in } \Omega
$$

and $u$ is subject to appropriate boundary conditions with the differential operator defined as

$$
\mathcal{L}=\sum_{m=0}^{n} a_{m}(-\Delta)^{m},
$$

where $\Delta$ is the Laplacian and $a_{m} \in L^{\infty}(\Omega), m=0,1, \cdots, n$ with $n$ being a positive integer. For simplicity, we assume that $\Omega=[0,1]^{d} \in \mathbb{R}^{d}, d=1,2,3$ and $a_{m}$ are constants in the following discussions.

The operator $\mathcal{L}$ in the general equation 2.1 covers many high-order differential operators arising in sciences and engineering. In particular:

- For $n=1, a_{0}=0, a_{1}=1, \mathcal{L}=-\Delta$ and 2.1 reduces to the Laplacian (or linearized Allen-Cahn [42]) eigenvalue problem.

- For $n=2, a_{0}=a_{1}=0, a_{2}=1, \mathcal{L}=\Delta^{2}$ and 2.1 becomes the biharmonic eigenvalue problem.

- For $n=2, a_{0}=0, a_{1}=1, a_{2}=1, \mathcal{L}=\Delta^{2}-\Delta$ and 2.1 is the linearized CahnHilliard eigenvalue problem of fourth order [43].

- For $n=2, a_{0}=1, a_{1}=-2, a_{2}=1, \mathcal{L}=(1+\Delta)^{2}$ and (2.1) is the linearized Swift-Hohenberg eigenvalue problem 44 .

- For $n=3, a_{0}=a_{1}=0, a_{2}=1, a_{3}=1, \mathcal{L}=-\Delta\left(\Delta^{2}-\Delta\right)$ and (2.1) is the linearized Cahn-Hilliard eigenvalue problem of sixth order [45].

- For $n=3, a_{0}=0, a_{1}=1, a_{2}=-2, a_{3}=1, \mathcal{L}=-\Delta(1+\Delta)^{2}$ and (2.1) is the linearized phase-field crystal eigenvalue problem [46].

We focus on the interfacial energy operator for the phase-field models listed. We chose this eigenvalue approximation as the linearized bulk energy contribution can vary orders of magnitude and of sign, making a general analysis beyond the scope of the present work. The differential eigenvalue problem (2.1) with constant coefficients $a_{m}, m=$ $0,1, \cdots, n$, has the following eigenpairs $\left(\lambda_{j}, u_{j}\right), j=1,2, \cdots$ : 
For 1D:

$$
\begin{aligned}
& \lambda_{j}=a_{n}\left(j^{2} \pi^{2}\right)^{n}+a_{n-1}\left(j^{2} \pi^{2}\right)^{n-1}+\cdots+a_{1}\left(j^{2} \pi^{2}\right)^{1}+a_{0}, \\
& u_{j}=C_{1} \sin (j \pi x)+C_{2} \cos (j \pi x) .
\end{aligned}
$$

For 2D:

$$
\begin{aligned}
& \lambda_{j l}=a_{n}\left(\left(j^{2}+l^{2}\right) \pi^{2}\right)^{n}+\cdots+a_{1}\left(\left(j^{2}+l^{2}\right) \pi^{2}\right)^{1}+a_{0}, \\
& u_{j l}=C_{1} \sin (j \pi x) \sin (l \pi y)+C_{2} \cos (j \pi x) \cos (l \pi y) .
\end{aligned}
$$

For 3D:

$$
\begin{aligned}
& \lambda_{j l q}=a_{n}\left(\left(j^{2}+l^{2}+q^{2}\right) \pi^{2}\right)^{n}+\cdots+a_{1}\left(\left(j^{2}+l^{2}+q^{2}\right) \pi^{2}\right)^{1}+a_{0}, \\
& u_{j l q}=C_{1} \sin (j \pi x) \sin (l \pi y) \sin (q \pi z)+C_{2} \cos (j \pi x) \cos (l \pi y) \cos (q \pi z) .
\end{aligned}
$$

Here the constants $C_{1}, C_{2} \in \mathbb{C}$ are determined by the boundary conditions. If we impose homogeneous Dirichlet or Neumann boundary conditions, then we obtain that $C_{2}=0$ or $C_{1}=0$, respectively. Once one constant is determined, the other constant can be determined by normalization, that is, to normalize $u$ such that $(u, u)_{L^{2}(\Omega)}=1$ where $(\cdot, \cdot)_{L^{2}(\Omega)}$ denotes the inner-product in $L^{2}(\Omega)$. In this paper, we consider homogeneous Dirichlet boundary conditions for the biharmonic eigenvalue problem (the case of a simply supported plate) and periodic boundary conditions for other eigenvalue problems.

\section{Mixed formulations}

To motivate the presentation of the mixed formulation for any $n$, we start with $n=2, a_{0}=a_{1}=0, a_{2}=1$ which (2.1) becomes the biharmonic eigenvalue problem. In this case, the differential equation (2.1) can be recast in a mixed form as a system of equation of second-order

$$
-\Delta u=\mu \quad \text { and } \quad-\Delta \mu=\lambda u
$$

and both $u$ and $\mu$ are subject to appropriate boundary conditions. This system of equations is referred to as problem with two unknown fields; see for example [3, 20, 47. This new auxiliary parameter has physical meanings. For example, in structural mechanics, the new unknown $\mu$ represents the bending moment [48, while in fluid dynamics, when the Stokes equations for viscous steady flow is transformed using stream function this represents the vorticity [49.

If the domain $\Omega$ has smooth boundary or it is convex polygonal domain, then the eigenvalue problem (3.1) has infinitely many solutions $\left(\lambda_{j},\left(\mu_{j}, u_{j}\right)\right)$ such that $50-52$ ]

$$
\begin{aligned}
0<\lambda_{1} & \leq \lambda_{2} \leq \lambda_{3} \leq \cdots<\infty, \\
\mu_{j} & =-\Delta u_{j}, \quad \forall j, k \geq 1, \\
\left(u_{j}, u_{k}\right)_{L^{2}(\Omega)} & =\delta_{j k}, \quad \forall j, \quad \text {, }
\end{aligned}
$$

with the Kronecker delta defined as $\delta_{j k}=1$ when $j=k$ and zero otherwise. Here, the eigenfunctions $u_{j}$ are orthonormal in $L^{2}(\Omega)$.

We denote by eigenpairs the set of formed by each eigenvalue and its corresponding set of eigenfunctions. In general there are $n$ eigenfuctions for each eigenvalue of the mixed system. If $\left(\lambda_{j},\left(\mu_{j}, u_{j}\right)\right)$ is an eigenpair of (3.1), then $\left(\lambda_{j}, u_{j}\right)$ is an eigenpair of (2.1) and $\mu=-\Delta u$. Hence the regularity of $\left(\mu_{j}, u_{j}\right)$ can be inferred from the regularity properties of problem (2.1); see for example 49. 
For an arbitrary positive integer $n$, we set

$$
\psi^{m}=-\Delta \psi^{m-1}
$$

for $m=1,2, \cdots, n-1$ with $\psi^{0}=u$. These auxiliary unknowns allow us to recast the differential equation (2.1) into the mixed form as a system of equations of second-order

$$
\begin{aligned}
-\Delta \psi^{m-1}-\psi^{m} & =0, m=1,2, \cdots, n-1, \\
-a_{n} \Delta \psi^{n-1}+\sum_{m=0}^{n-1} a_{m} \psi^{m} & =\lambda u .
\end{aligned}
$$

Similarly, we expect that if $\left(\lambda_{j},\left(\psi_{j}^{1}, \psi_{j}^{2}, \cdots, \psi_{j}^{n-1}, u_{j}\right)\right)$ is an eigenpair of (3.4), then $\left(\lambda_{j}, u_{j}\right)$ is an eigenpair of 2.1 with $\psi_{j}^{m}=-\Delta \psi_{j}^{m-1}$ as in 3.3 . The regularity of $\left(\psi_{j}^{1}, \psi_{j}^{2}, \cdots, \psi_{j}^{n-1}, u_{j}\right)$ can be inferred from the regularity properties of problem (2.1) and we assume sufficient regularity of the problem (2.1).

Now, we present the mixed variational formulation for (2.1) at the continuous level. We denote the standard $L^{2}(\Omega)$-norm as $\|\cdot\|_{0, \Omega} \equiv\|\cdot\| \equiv\|\cdot\|_{L^{2}(\Omega)}$. We adopt the standard Sobolev spaces of integer index $s, H^{s}(\Omega)$ and $H_{0}^{s}(\Omega)$, equipped with the norm $\|\cdot\|_{s, \Omega} \equiv\|\cdot\|_{H^{s}(\Omega)} ;$ see [47, 53].

We define the bilinear forms

$$
a(v, w)=\int_{\Omega} \nabla v \cdot \nabla w \mathrm{~d} x, \quad b(v, w)=\int_{\Omega} v w \mathrm{~d} x
$$

where $\nabla$ is the gradient operator. The bilinear forms $a(\cdot, \cdot)$ and $b(\cdot, \cdot)$ are usually referred as stiffness and mass, respectively. These bilinear forms can be written alternatively as

$$
a(v, w)=(\nabla v, \nabla w)_{L^{2}(\Omega)}, \quad b(v, w)=(v, w)_{L^{2}(\Omega)} .
$$

At the continuous level, for simplicity, we assume that the differential equation 2.1 is subject to simply supported boundary conditions

$$
u=\Delta u=\Delta^{2} u=\cdots=\Delta^{n-1} u=0 .
$$

The mixed formulations for (2.1) or (3.4) is: Find the eigenpairs $\left(\lambda,\left(\psi^{1}, \psi^{2}, \cdots, \psi^{n-1}, u\right)\right)$ with $\lambda \in \mathbb{R}$ and $\psi^{m}, u \in H_{0}^{1}(\Omega), m=1,2, \cdots, n-1$, satisfying

$$
\begin{aligned}
a\left(\psi^{m-1}, w^{m}\right)-b\left(\psi^{m}, w^{m}\right) & =0, m=1,2, \cdots, n-1, \quad \forall w^{m} \in H_{0}^{1}(\Omega), \\
a_{n} a\left(\psi^{n-1}, v\right)+\sum_{m=0}^{n-1} a_{m} b\left(\psi^{m}, v\right) & =\lambda b(u, v), \quad \forall v \in H_{0}^{1}(\Omega) .
\end{aligned}
$$

Remark 1. One can also consider other boundary conditions. For example, for $n=$ $2, a_{0}=a_{1}=0, a_{2}=1$, with homogeneous Dirichlet boundary conditions

$$
u=\frac{\partial u}{\partial \nu}=0 \quad \text { on } \quad \partial \Omega,
$$


the mixed variational formulation of the corresponding 2.1 is: Find the eigenpairs $\left(\lambda,\left(\psi^{1}, u\right)\right)$ with $\lambda \in \mathbb{R}, \psi^{1} \in H^{1}(\Omega)$, and $u \in H_{0}^{1}(\Omega)$ satisfying

$$
\begin{aligned}
a(u, w)-b\left(\psi^{1}, w\right) & =0, \quad \forall w \in H^{1}(\Omega), \\
a\left(\psi^{1}, v\right) & =\lambda b(u, v), \quad \forall v \in H_{0}^{1}(\Omega) .
\end{aligned}
$$

Herein, $\nu$ denotes the outward unit normal to the boundary $\partial \Omega$. We refer the readers to [49] for details.

\subsection{Mixed formulation at discrete level}

Let $\mathcal{T}_{h}$ be a partition of $\Omega$ into non-overlapping tensor-product mesh elements and let $K \in \mathcal{T}_{h}$ be a generic element. At the discrete level, we specify a finite dimensional approximation space $V_{h}^{p} \subset H_{0}^{1}(\Omega)$ where $V_{h}^{p}=\operatorname{span}\left\{\phi_{a}^{p}\right\}$ is the span of the B-spline or Lagrange (for FEM) basis functions $\phi_{a}^{p}$ of order $p$. Consequently, the mixed isogeometric analysis (or FEM) of 2.1) with simply supported boundary conditions (3.7) is to seek $\lambda^{h} \in \mathbb{R}$ and $\psi_{h}^{m}, u_{h} \in V_{h}^{p}, m=1,2, \cdots, n-1$, satisfying

$$
\begin{aligned}
a\left(\psi_{h}^{m-1}, w_{h}^{m}\right)-b\left(\psi_{h}^{m}, w_{h}^{m}\right) & =0, m=1,2, \cdots, n-1, \quad \forall w_{h}^{m} \in V_{h}^{p}, \\
a_{n} a\left(\psi_{h}^{n-1}, v_{h}\right)+\sum_{m=0}^{n-1} a_{m} b\left(\psi_{h}^{m}, v_{h}\right) & =\lambda^{h} b\left(u_{h}, v_{h}\right), \quad \forall v_{h} \in V_{h}^{p} .
\end{aligned}
$$

The definition of the B-spline basis functions in one dimension is as follows. Let $X=\left\{x_{0}, x_{1}, \cdots, x_{m}\right\}$ be a knot vector with knots $x_{j}$, that is, a nondecreasing sequence of real numbers which are called knots. The $j$-th B-spline basis function of degree $p$, denoted as $\theta_{p}^{j}(x)$, is defined as $[54,55]$

$$
\begin{aligned}
& \theta_{0}^{j}(x)= \begin{cases}1, & \text { if } x_{j} \leq x<x_{j+1} \\
0, & \text { otherwise }\end{cases} \\
& \theta_{p}^{j}(x)=\frac{x-x_{j}}{x_{j+p}-x_{j}} \theta_{p-1}^{j}(x)+\frac{x_{j+p+1}-x}{x_{j+p+1}-x_{j+1}} \theta_{p-1}^{j+1}(x) .
\end{aligned}
$$

In this paper, for isogeometric analysis, we utilize the B-splines on uniform tensorproduct meshes with non-repeating knots, that is, the B-splines with maximum continuity on uniform meshes, while for finite element method, we utilize the standard Lagrange basis functions. We approximate the eigenfunctions as a linear combination of the Bspline (or Lagrange) basis functions and substitute all the basis functions for $V_{h}^{p}$ in (3.11). This leads to a matrix eigenvalue problem, which is then solved numerically. We give more details of the structures of matrix eigenvalue problem in the following.

\subsection{Quadrature rules}

In practice, we evaluate the integrals involved in the bilinear forms $a(\cdot, \cdot)$ and $b(\cdot, \cdot)$ numerically, that is using quadrature rules. On a reference element $\hat{K}$, a quadrature rule is of the form

$$
\int_{\hat{K}} \hat{f}(\hat{\boldsymbol{x}}) \mathrm{d} \hat{\boldsymbol{x}} \approx \sum_{l=1}^{N_{q}} \hat{\varpi}_{l} \hat{f}\left(\hat{n}_{l}\right),
$$


where $\hat{\varpi}_{l}$ are the weights, $\hat{n}_{l}$ are the nodes (quadrature points), and $N_{q}$ is the number of quadrature points. For each element $K$, we assume that there is an invertible map $\sigma$ such that $K=\sigma(\hat{K})$, which leads to the correspondence between the functions on $K$ and $\hat{K}$. Assuming $J_{K}$ is the corresponding Jacobian of the mapping, (3.13) induces a quadrature rule over the element $K$ given by

$$
\int_{K} f(\boldsymbol{x}) \mathrm{d} \boldsymbol{x} \approx \sum_{l=1}^{N_{q}} \varpi_{l, K} f\left(n_{l, K}\right),
$$

where $\varpi_{l, K}=\operatorname{det}\left(J_{K}\right) \hat{\varpi}_{l}$ and $n_{l, K}=\sigma\left(\hat{n}_{l}\right)$. For simplicity, we denote by $G_{l}$ the $l$-point Gauss-Legendre quadrature rule, by $L_{l}$ the $l$-point Gauss-Lobatto quadrature rule, by $R_{l}$ the $l$-point Gauss-Radau quadrature rule, and by $O_{p}$ the optimal blending scheme for the $p$-th order isogeometric analysis with maximum continuity. In one dimension on the reference element or under affine mappings, $G_{l}, L_{l}$, and $R_{l}$ fully integrate polynomials of order $2 l-1,2 l-3$, and $2 l-2$, respectively $56-58$.

Applying quadrature rules to (3.11), we obtain the approximate form

$$
\begin{aligned}
a_{h}\left(\psi_{h}^{m-1}, w_{h}^{m}\right)-b_{h}\left(\psi_{h}^{m}, w_{h}^{m}\right) & =0, m=1,2, \cdots, n-1, \quad \forall w_{h}^{m} \in V_{h}^{p}, \\
a_{n} a_{h}\left(\psi_{h}^{n-1}, v_{h}\right)+\sum_{m=0}^{n-1} a_{m} b_{h}\left(\psi_{h}^{m}, v_{h}\right) & =\tilde{\lambda}^{h} b_{h}\left(u_{h}, v_{h}\right), \quad \forall v_{h} \in V_{h}^{p},
\end{aligned}
$$

where for $w, v \in V_{h}^{p}$

$$
a_{h}(w, v)=\sum_{K \in \mathcal{T}_{h}} \sum_{l=1}^{N_{q}^{1}} \varpi_{l, K}^{(1)} \nabla w\left(n_{l, K}^{(1)}\right) \cdot \nabla v\left(n_{l, K}^{(1)}\right)
$$

and

$$
b_{h}(w, v)=\sum_{K \in \mathcal{T}_{h}} \sum_{l=1}^{N_{q}^{2}} \varpi_{l, K}^{(2)} w\left(n_{l, K}^{(2)}\right) v\left(n_{l, K}^{(2)}\right)
$$

with $\left\{\varpi_{l, K}^{(1)}, n_{l, K}^{(1)}\right\}$ and $\left\{\varpi_{l, K}^{(2)}, n_{l, K}^{(2)}\right\}$ specifying two (possibly different) quadrature rules. Using quadrature rules, we can write the matrix eigenvalue problem as

$$
\left[\begin{array}{ccccc}
\boldsymbol{K} & -\boldsymbol{M} & \mathbf{0} & \mathbf{0} & \mathbf{0} \\
\mathbf{0} & \boldsymbol{K} & -\boldsymbol{M} & \mathbf{0} & \mathbf{0} \\
\vdots & \vdots & \vdots & \ddots & \vdots \\
\mathbf{0} & \mathbf{0} & \cdots & \boldsymbol{K} & -\boldsymbol{M} \\
a_{0} \boldsymbol{M} & a_{1} \boldsymbol{M} & \cdots & a_{n-2} \boldsymbol{M} & a_{n-1} \boldsymbol{M}+a_{n} \boldsymbol{K}
\end{array}\right]\left[\begin{array}{c}
\boldsymbol{U} \\
\boldsymbol{\Psi}^{1} \\
\vdots \\
\boldsymbol{\Psi}^{n-2} \\
\boldsymbol{\Psi}^{n-1}
\end{array}\right]=\tilde{\lambda}^{h}\left[\begin{array}{cc}
\mathbf{0} & \mathbf{0} \\
\boldsymbol{M} & \mathbf{0}
\end{array}\right]\left[\begin{array}{c}
\boldsymbol{U} \\
\boldsymbol{\Psi}^{1} \\
\vdots \\
\boldsymbol{\Psi}^{n-2} \\
\boldsymbol{\Psi}^{n-1}
\end{array}\right]
$$

where $\boldsymbol{K}_{a b}=a_{h}\left(\phi_{a}^{p}, \phi_{b}^{p}\right), \boldsymbol{M}_{a b}=b_{h}\left(\phi_{a}^{p}, \phi_{b}^{p}\right)$, and $\boldsymbol{U}, \boldsymbol{\Psi}^{j}, j=1, \cdots, n-1$ are the corresponding representation of the eigenvector as the coefficients of the basis functions. Similar to the standard second-order eigenvalue problem, $\boldsymbol{K}$ and $\boldsymbol{M}$ are referred to as the stiffness and mass matrices resulting from (3.5), respectively. This matrix eigenvalue problem $(3.18)$ has a similar structure with the one obtained by hybrid high-order discretization; see [59, Eqn. 3.13]. 
The optimally-blended rules are developed and analyzed for isogeometric analysis in [1, 2, 41, 60] for $p \leq 7$ and generalized to arbitrary order $p$ in [61. We define the blending quadrature rule $\mathcal{Q}_{\tau}$ as

$$
\int_{K} f(\boldsymbol{x}) \mathrm{d} \boldsymbol{x} \approx \mathcal{Q}_{\tau}(f)=\tau \mathcal{Q}_{1}(f)+(1-\tau) \mathcal{Q}_{2}(f),
$$

where $\tau$ is the blending parameter and $\mathcal{Q}_{1}, \mathcal{Q}_{2}$ are different quadrature rules. We denote the following blendings of Gauss-Legendre rule $G_{p}, G_{p+1}$ and Gauss-Lobatto rule $L_{p+1}$ as

$$
\tau_{g g} G_{p+1}+\left(1-\tau_{g g}\right) G_{p}, \quad \tau_{g l} G_{p+1}+\left(1-\tau_{g l}\right) L_{p+1},
$$

where $\tau_{g g}$ and $\tau_{g l}$ are blending parameters.

\begin{tabular}{|c||c|c|c|c|}
\hline$p$ & 1 & 2 & 3 & 4 \\
\hline$\tau_{g g}$ & 2 & 2 & $\frac{13}{3}$ & 22 \\
\hline$\tau_{g l}$ & $\frac{1}{2}$ & $\frac{1}{3}$ & $-\frac{3}{2}$ & $-\frac{79}{5}$ \\
\hline
\end{tabular}

Table 1: Optimal blending parameters.

Table 1 shows the optimal blending parameters for $p \leq 4$; see also [2, 61. For optimal blending parameters of higher order $p$ and blending among other quadrature rules, we refer to 61. These optimally-blended quadrature rules improve spectrum errors significantly, which we show in the next section.

\section{Eigenvalue errors}

In this section, following the eigenvalue error estimates established in 1, 2, 61 for the second-order Laplacian eigenvalue problem, we derive a priori eigenvalue error estimates for (3.18), which can be viewed as a generalization to the $2 n$-order differential eigenvalue problems. The mixed formulation delivers the optimal error convergence rates for the biharmonic eigenvalue problem.

Following the structure in 61, we seek an approximation of eigenfunction $u_{h}$ in the form

$$
\sum_{j=0}^{N} U^{j} \theta_{p}^{j}(x),
$$

where $U^{j}$ are the unknown coefficients which corresponds to the the $p$-th order polynomial approximation which are to be determined, that is the component of the unknown vector $\boldsymbol{U}$ in (3.18). Using the Bloch wave assumption [62, we write

$$
U^{j}=e^{i j \omega h}
$$

where $i^{2}=-1$ and $\omega$ is an approximated frequency. In the view of auxiliary fields (3.3), we denote $\psi_{h}^{m}=u_{h}$ and $\boldsymbol{\Psi}^{m}=\boldsymbol{U}$ when $m=0$. In the Bloch wave assumption $(4.2), j h$ 
resembles the spatial variable $x$. Using the auxiliary fields defined (3.3), this allows us further assume Bloch wave solutions for their derivatives, that is

$$
\Psi^{m, j}=\omega^{2 m} e^{i j \omega h}, m=0,1,2, \cdots, n-1,
$$

where $\Psi^{m, j}$ is the component of the unknown vector $\Psi^{m}$ in 3.18$)$. For $m=0$, equation (4.3) simplifies to (4.2).

The $C^{p-1} \mathrm{~B}$-spline basis function $\theta_{p}^{j}$ has a support over $p+1$ elements. Thus, for $m=0,1,2, \cdots, n-1$, we have

$$
\begin{aligned}
& a_{h}\left(\psi_{h}^{m}, \theta_{p}^{j}\right)=a_{h}\left(\sum_{k,|k-j| \leq p} \Psi_{p}^{m, j} \theta_{p}^{k}, \theta_{p}^{j}\right)=A_{p} \Psi_{p}^{m} / h, \\
& b_{h}\left(\psi_{h}^{m}, \theta_{p}^{j}\right)=b_{h}\left(\sum_{k,|k-j| \leq p} \Psi_{p}^{m, j} \theta_{p}^{k}, \theta_{p}^{j}\right)=B_{p} \Psi_{p}^{m} h,
\end{aligned}
$$

where $a_{h}(\cdot, \cdot), b_{h}(\cdot, \cdot)$ approximate (or exactly-integrate for appropriate quadrature rules) bilinear forms and

$$
\begin{aligned}
\Psi_{p}^{m} & =\left[\begin{array}{llllllll}
\Psi_{p}^{m, j-p} & \Psi_{p}^{m, j-p+1} & \cdots & \Psi_{p}^{m, j} & \cdots & \Psi_{p}^{m, j+p-1} & \Psi_{p}^{m, j+p}
\end{array}\right]^{T}, \\
A_{p} & =\left[\begin{array}{llllllll}
A_{p}^{j-p} & A_{p}^{j-p+1} & \cdots & A_{p}^{j} & \cdots & A_{p}^{j+p-1} & A_{p}^{j+p}
\end{array}\right], \\
B_{p} & =\left[\begin{array}{lllllll}
B_{p}^{j-p} & B_{p}^{j-p+1} & \cdots & B_{p}^{j} & \cdots & B_{p}^{j+p-1} & B_{p}^{j+p}
\end{array}\right],
\end{aligned}
$$

with

$$
A_{p}^{j-k}=a_{h}\left(\theta_{p}^{j-k}, \theta_{p}^{j}\right) h, \quad B_{p}^{j-k}=b_{h}\left(\theta_{p}^{j-k}, \theta_{p}^{j}\right) / h
$$

for $k=p, p-1, \cdots,-p$. For $m=0$, we also denote $\Psi_{p}^{m}=U_{p}$ with

$$
U_{p}=\left[\begin{array}{lllllll}
U_{p}^{j-p} & U_{p}^{j-p+1} & \cdots & U_{p}^{j} & \cdots & U_{p}^{j+p-1} & U_{p}^{j+p}
\end{array}\right]^{T} .
$$

The symmetry of the B-spline basis functions (on uniform meshes and away from the boundaries) further implies that

$$
A_{p}^{j-k}=A_{p}^{j+k}, \quad B_{p}^{j-k}=B_{p}^{j+k} .
$$

Thus, using this symmetry, Euler's formula, Bloch wave assumptions 4.2 and 4.3 , we can deduce the following

$$
\begin{aligned}
& a_{h}\left(\psi_{h}^{m}, \theta_{p}^{j}\right)=A_{p} \Psi_{p}^{m} / h=\omega^{2 m}\left(A_{p}^{j}+2 \sum_{k=1}^{p} A_{p}^{j+k} \cos (k \omega h)\right) e^{i j \omega h} / h=\omega^{2 m} A_{p} U_{p} / h, \\
& b_{h}\left(\psi_{h}^{m}, \theta_{p}^{j}\right)=B_{p} \Psi_{p}^{m} h=\omega^{2 m}\left(B_{p}^{j}+2 \sum_{k=1}^{p} B_{p}^{j+k} \cos (k \omega h)\right) e^{i j \omega h} h=\omega^{2 m} B_{p} U_{p} h .
\end{aligned}
$$

Lemma 1. Denote $\Lambda=\omega h$. For any positive integer $p$, denoting

$$
C_{p+2}=2(-1)^{p}\left(\sum_{k=1}^{p} \frac{k^{2 p+4}}{(2 p+4) !} A_{p}^{j+k}+\frac{k^{2 p+2}}{(2 p+2) !} B_{p}^{j+k}\right),
$$


there holds

$$
\frac{A_{p} U_{p}}{B_{p} U_{p}}=\Lambda^{2}+C_{p+2} \Lambda^{2 p+4}+\mathcal{O}\left(\Lambda^{2 p+6}\right),
$$

when $a_{h}(\cdot, \cdot)$ and $b_{h}(\cdot, \cdot)$ in (4.6) are approximated using the optimally blended quadrature rules.

Proof. We omit the proof as the identity is proved using the same arguments in the proof of Lemma 6 supplied with Lemma 7 in [61].

Theorem 1. Assuming $a_{h}(\cdot, \cdot)$ and $b_{h}(\cdot, \cdot)$ in (4.6) are approximated using the optimally blended quadrature rules, there holds

$$
\tilde{\lambda}^{h}-\sum_{k=0}^{n} a_{k} \omega^{2 k}=\left(C_{p+2} \sum_{k=1}^{n} a_{k} \omega^{2 k}\right)(\omega h)^{2 p+2}+\mathcal{O}(h)^{2 p+4} .
$$

Proof. Let $\theta_{p}^{j}$ be a test function for each equation in 3.15). We represent the approximated eigenfunctions $\psi_{h}^{m}, m=0,1, \cdots, n-1$, in the the same way as 4.1. Substituting all these terms into 3.15 , we obtain

$$
\begin{aligned}
A_{p} \Psi_{p}^{m-1} / h-B_{p} \Psi_{p}^{m} h & =0, m=1,2, \cdots, n-1, \\
a_{n} A_{p} \Psi_{p}^{n-1} / h+\sum_{m=0}^{n-1} a_{m} B_{p} \Psi_{p}^{m} h & =\tilde{\lambda}^{h} B_{p} \Psi_{p}^{0} h .
\end{aligned}
$$

After substituting the first equation into the second one, simple manipulations yield

$$
\sum_{m=1}^{n} a_{m} A_{p} \Psi_{p}^{m-1}=\left(\tilde{\lambda}^{h}-a_{0}\right) B_{p} \Psi_{p}^{0} h^{2}
$$

which, by using (4.9), is further simplified as

$$
\sum_{m=1}^{n} a_{m} \omega^{2 m-2} A_{p} U_{p}=\left(\tilde{\lambda}^{h}-a_{0}\right) B_{p} U_{p} h^{2} .
$$

Applying Lemma 1, we have

$$
\frac{\left(\tilde{\lambda}^{h}-a_{0}\right) h^{2}}{\sum_{m=1}^{n} a_{m} \omega^{2 m-2}}=\frac{A_{p} U_{p}}{B_{p} U_{p}}=(\omega h)^{2}+C_{p+2}(\omega h)^{2 p+4}+\mathcal{O}(\omega h)^{2 p+6},
$$

which is further simplified to

$$
\tilde{\lambda}^{h}-a_{0}=\sum_{m=1}^{n} a_{m} \omega^{2 m}+\left(C_{p+2} \sum_{m=1}^{n} a_{m} \omega^{2 m}\right)(\omega h)^{2 p+2}+\mathcal{O}\left(h^{2 p+4}\right) .
$$

143 Rewriting the equation completes the proof.

Remark 2. In the case where the true eigenvalue can be rewritten in form of $\lambda=$ $\sum_{k=0}^{n} a_{k} \omega^{2 k}$ as in (2.3), (2.4), and (2.5), the mixed formulations with optimally-blended 
quadrature rules are applied, the method retains its optimal rates $\left|\lambda_{h}-\lambda\right| \approx \mathcal{O}\left(h^{2 p}\right)$. This theorem establishes that the mixed formulation for $2 n$-order differential eigenvalue maintains the theoretical findings established for the approximation method of the secondorder Laplacian eigenvalue problem. For the generalization to multiple dimensions, we refer to [61] for the second-order Laplacian eigenvalue problem.

\section{Numerical experiments}

In this section, we present $1 \mathrm{D}, 2 \mathrm{D}$, and 3D numerical results which cover the spectral approximations of the biharmonic, Cahn-Hilliard, Swift-Hohenberg, and phase-field crystal operators. We limit our studies to simple geometrical domains and uniform meshes to focus our attention on the numerical aspects of the problem. We utilize both mixed isogeometric and finite elements with $p=1,2,3,4$. For our simulations, we consider the unitary domain $\Omega=[0,1]^{d}$. The exact solutions of 2.1 are given in $(2.3)-2.5$ with the parameters specified in Section 2

First of all, we present the matrix eigenvalue problems associated with the operators described above, which are easily obtained from the general representation (3.18). In particular, with slight abuse of notation, we have

$$
\left[\begin{array}{cc}
\boldsymbol{K} & -\boldsymbol{M} \\
\mathbf{0} & \boldsymbol{K}
\end{array}\right]\left[\begin{array}{c}
\boldsymbol{U} \\
\boldsymbol{\Psi}^{1}
\end{array}\right]=\tilde{\lambda}^{h}\left[\begin{array}{cc}
\mathbf{0} & \mathbf{0} \\
\boldsymbol{M} & \mathbf{0}
\end{array}\right]\left[\begin{array}{c}
\boldsymbol{U} \\
\boldsymbol{\Psi}^{1}
\end{array}\right]
$$

for the biharmonic eigenvalue problem,

$$
\left[\begin{array}{cc}
\boldsymbol{K} & -\boldsymbol{M} \\
\mathbf{0} & \boldsymbol{K}+\boldsymbol{M}
\end{array}\right]\left[\begin{array}{c}
\boldsymbol{U} \\
\boldsymbol{\Psi}^{1}
\end{array}\right]=\tilde{\lambda}^{h}\left[\begin{array}{cc}
\mathbf{0} & \mathbf{0} \\
\boldsymbol{M} & \mathbf{0}
\end{array}\right]\left[\begin{array}{c}
\boldsymbol{U} \\
\boldsymbol{\Psi}^{1}
\end{array}\right]
$$

for the Cahn-Hilliard eigenvalue problem,

$$
\left[\begin{array}{lc}
\boldsymbol{K} & -\boldsymbol{M} \\
\boldsymbol{M} & \boldsymbol{K}-2 \boldsymbol{M}
\end{array}\right]\left[\begin{array}{c}
\boldsymbol{U} \\
\boldsymbol{\Psi}^{1}
\end{array}\right]=\tilde{\lambda}^{h}\left[\begin{array}{cc}
\mathbf{0} & \mathbf{0} \\
\boldsymbol{M} & \mathbf{0}
\end{array}\right]\left[\begin{array}{c}
\boldsymbol{U} \\
\boldsymbol{\Psi}^{1}
\end{array}\right]
$$

for the Swift-Hohenberg eigenvalue problem, and

$$
\left[\begin{array}{ccc}
\boldsymbol{K} & -\boldsymbol{M} & \mathbf{0} \\
\mathbf{0} & \boldsymbol{K} & -\boldsymbol{M} \\
\mathbf{0} & \boldsymbol{M} & \boldsymbol{K}-2 \boldsymbol{M}
\end{array}\right]\left[\begin{array}{c}
\boldsymbol{U} \\
\boldsymbol{\Psi}^{1} \\
\boldsymbol{\Psi}^{2}
\end{array}\right]=\tilde{\lambda}^{h}\left[\begin{array}{ccc}
\mathbf{0} & \mathbf{0} & \mathbf{0} \\
\mathbf{0} & \mathbf{0} & \mathbf{0} \\
\boldsymbol{M} & \mathbf{0} & \mathbf{0}
\end{array}\right]\left[\begin{array}{c}
\boldsymbol{U} \\
\boldsymbol{\Psi}^{1} \\
\boldsymbol{\Psi}^{2}
\end{array}\right]
$$

for the phase-field crystal eigenvalue problem, respectively. For the fourth-order differential eigenvalue problem, one can symmetrize the matrix on the left-hand side, that is, the matrix eigenvalue problem $(5.1)$ to $(5.3)$ are equivalent to

$$
\begin{gathered}
{\left[\begin{array}{cc}
\boldsymbol{K} & -\boldsymbol{M} \\
-\boldsymbol{M} & \boldsymbol{K}
\end{array}\right]\left[\begin{array}{c}
\boldsymbol{U} \\
\boldsymbol{\Psi}^{1}
\end{array}\right]=\left(\tilde{\lambda}^{h}-1\right)\left[\begin{array}{cc}
\mathbf{0} & \mathbf{0} \\
\boldsymbol{M} & \mathbf{0}
\end{array}\right]\left[\begin{array}{c}
\boldsymbol{U} \\
\boldsymbol{\Psi}^{1}
\end{array}\right],} \\
{\left[\begin{array}{cc}
\boldsymbol{K} & -\boldsymbol{M} \\
-\boldsymbol{M} & \boldsymbol{K}+\boldsymbol{M}
\end{array}\right]\left[\begin{array}{c}
\boldsymbol{U} \\
\boldsymbol{\Psi}^{1}
\end{array}\right]=\left(\tilde{\lambda}^{h}-1\right)\left[\begin{array}{cc}
\mathbf{0} & \mathbf{0} \\
\boldsymbol{M} & \mathbf{0}
\end{array}\right]\left[\begin{array}{c}
\boldsymbol{U} \\
\boldsymbol{\Psi}^{1}
\end{array}\right],}
\end{gathered}
$$

and

$$
\left[\begin{array}{cc}
\boldsymbol{K} & -\boldsymbol{M} \\
-\boldsymbol{M} & \boldsymbol{K}-2 \boldsymbol{M}
\end{array}\right]\left[\begin{array}{c}
\boldsymbol{U} \\
\boldsymbol{\Psi}^{1}
\end{array}\right]_{11}=\left(\tilde{\lambda}^{h}-2\right)\left[\begin{array}{cc}
\mathbf{0} & \mathbf{0} \\
\boldsymbol{M} & \mathbf{0}
\end{array}\right]\left[\begin{array}{l}
\boldsymbol{U} \\
\boldsymbol{\Psi}^{1}
\end{array}\right]
$$


respectively. Numerical experiments show that it takes less time to solve (5.5) to (5.7) than solving the original problems $(5.1)$ to $(5.3)$ that have non-symmetric matrices on the left-hand side. We use the symmetric systems to solve the differential eigenvalue problems numerically in the following experiments.

As the eigenvalues of $(2.1$ can be large, we present the relative eigenvalue errors, which for a $j$-th eigenvalue, is defined as

$$
e_{\lambda_{j}}=\frac{\left|\tilde{\lambda}_{j}^{h}-\lambda_{j}\right|}{\lambda_{j}}
$$

We start our accuracy and convergence studies in $1 \mathrm{D}$ and then extend them to multiple dimensions. Figure 1 shows the eigenvalue errors for isogeometric elements of maximum continuity for the biharmonic, Cahn-Hilliard and Swift-Hohenberg equations with homogeneous Dirichlet boundary conditions. The exact solutions are in form of (2.3) in 1D. Similar plots have been previously shown in 63 for approximate eigenvalues of second-order PDEs. As we can see in Figure 1, all three fourth-order operators have quite similar errors when the same boundary conditions are used; we observe a similar behaviour in multiple dimensions, as we show below.

The case of homogeneous Dirichlet boundary conditions has a particular importance for the biharmonic equation where it corresponds to the case of a simply supported plate. For the differential eigenvalue problems that describe the phase separation process, periodic boundary conditions are more relevant. In the following figures, we consider Dirichlet boundary conditions for the biharmonic equation and periodic boundary conditions for Cahn-Hilliard, Swift-Hohenberg, and phase-field crystal equations.

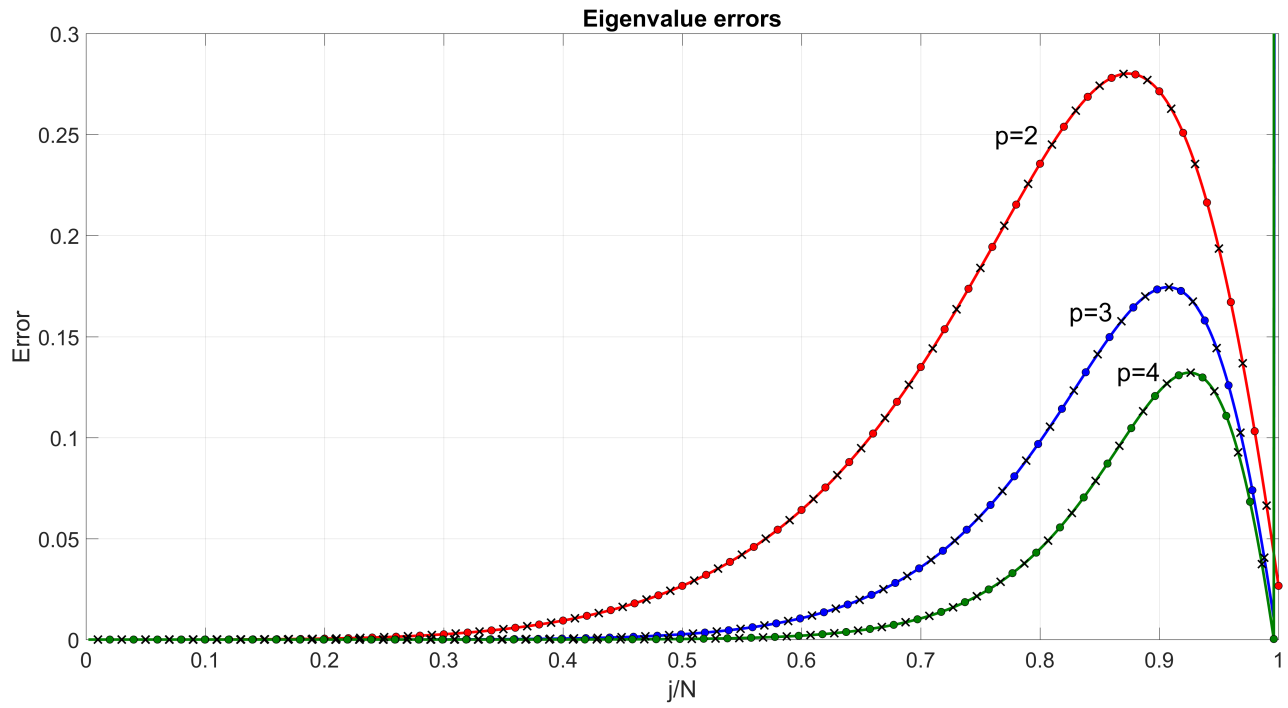

Figure 1: Relative approximation errors for quadratic, cubic and quartic isogeometric elements for the biharmonic (solid lines), Cahn-Hilliard (circles) and Swift-Hohenberg (crosses) equations in 1D.

Figure 2 shows the approximation errors for 1D Cahn-Hilliard and phase-field crystal operators with periodic boundary conditions for $p=2,3,4$. The Swift-Hohenberg spectra 
are similar to the Cahn-Hilliard results and are omitted for brevity. Once again, the spectral accuracy of the IGA discretizations improves with an increase in the polynomial order $p$. Due to the use of periodic boundary conditions, outlier modes (large spikes in the errors for $j / N$ close to one (high-frequencies) in the spectra of high-order isogeometric discretizations) are absent in this case. This is related to the fact that outliers are caused by the basis functions with support on the boundaries of the domain [64, which are absent in the periodic case.
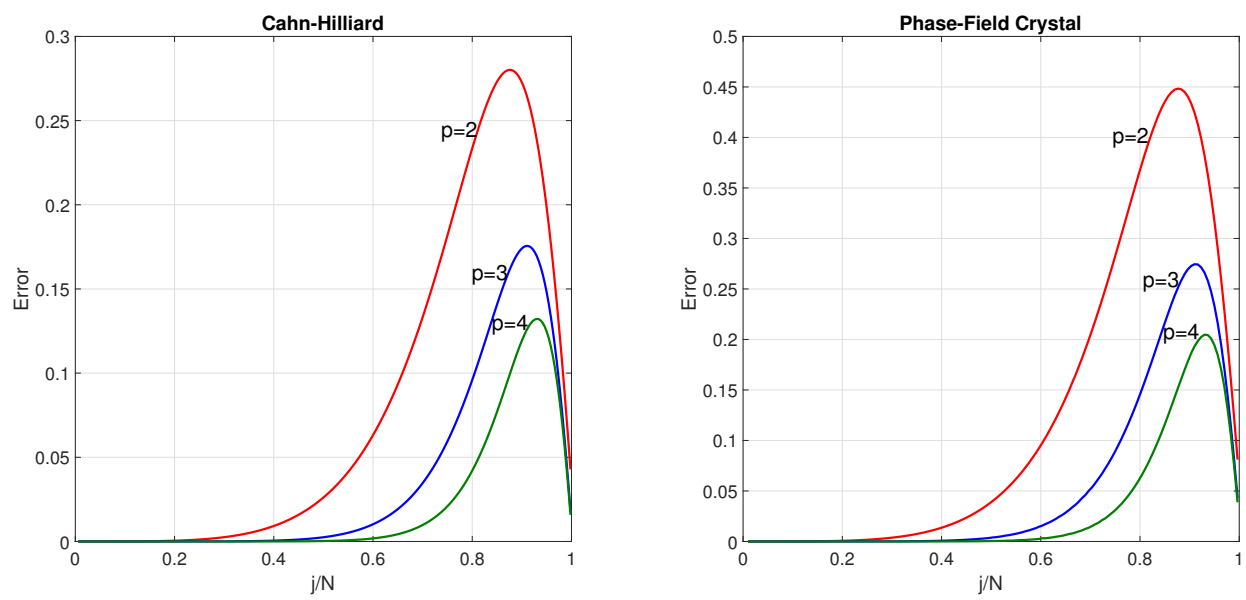

Figure 2: Relative approximation errors for Cahn-Hilliard and phase-field crystal operators with periodic boundary conditions for $p=2,3,4$. Note different Y scales across panels.

Figure 3 compares the eigenvalue and eigenfunction errors between the mixed $C^{0}$ finite elements and $C^{1}$ isogeometric elements. We observe branching of the finite element spectrum which is typical for high-order $C^{0}$ discretizations. Notably, B-spline basis functions of the highest $p-1$ continuity do not exhibit such branching patterns on uniform meshes. Similar to the standard (non-mixed) formulation, the stopping bands (the large spikes in the approximation errors in the middle of the spectra at the transition point between the acoustic and optical branches) are absent in mixed isogeometric discretizations.

In Figure 4, we compare the approximation errors for quadratic elements when using mixed isogeometric analysis with standard Gauss quadratures and optimally-blended rules (3.20). In this case, we also use Dirichlet boundary conditions for the biharmonic equation and periodic boundary conditions for Cahn-Hilliard and phase-field crystal equations. Despite this, all three cases exhibit similar behaviour. The optimally-blended rules lead to the convergence rates of order $2 p+2$ as predicted by the theory of subsection 3.4 .

Figure 5 compares the relative approximation errors for the 3D biharmonic eigenvalue problem. In the multidimensional plots shown below, the axes correspond to eigenvalue indices $j, l, q$ in (2.4) and 2.5. Again, using mixed isogeometric analysis with optimally-blended rules leads to smaller errors when compared to the fully-integrated case that employs standard Gauss quadratures.

Tables 2 to 5 show the first, second, fourth, and eighth eigenvalue errors when using 


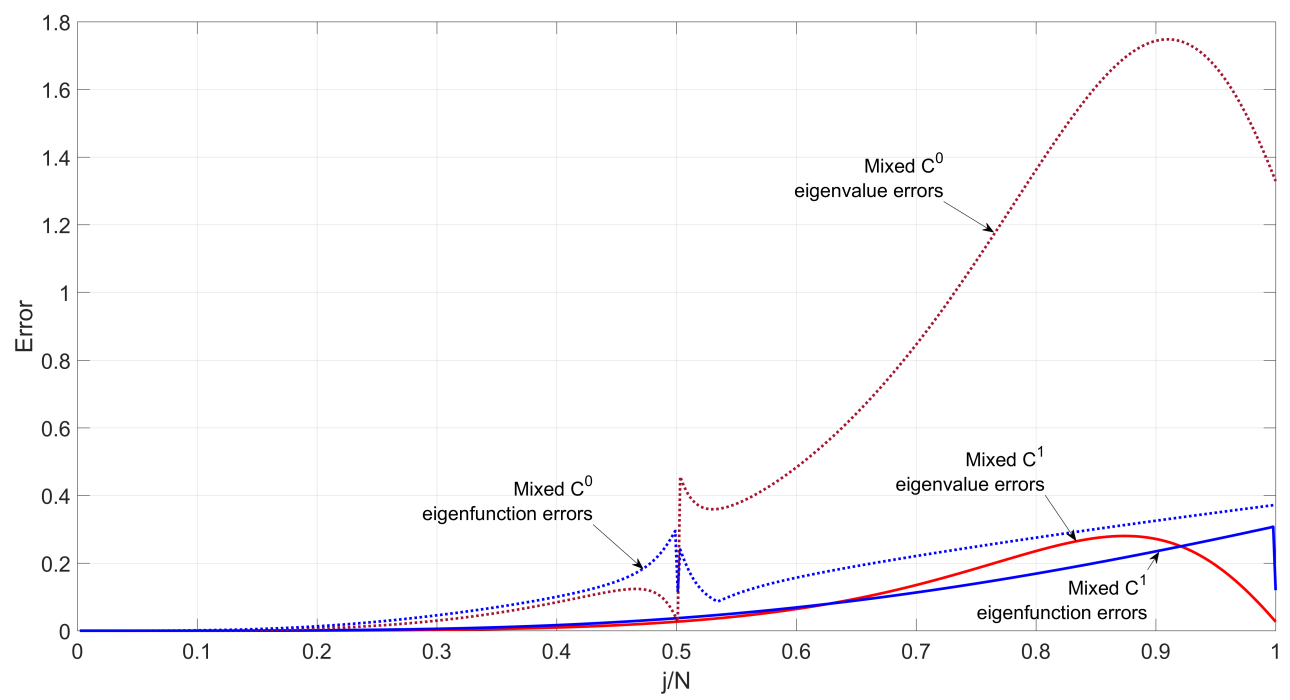

Figure 3: Relative eigenvalue and eigenfunction errors for quadratic $C^{0}$ and $C^{1}$ elements for the 1D biharmonic equation.
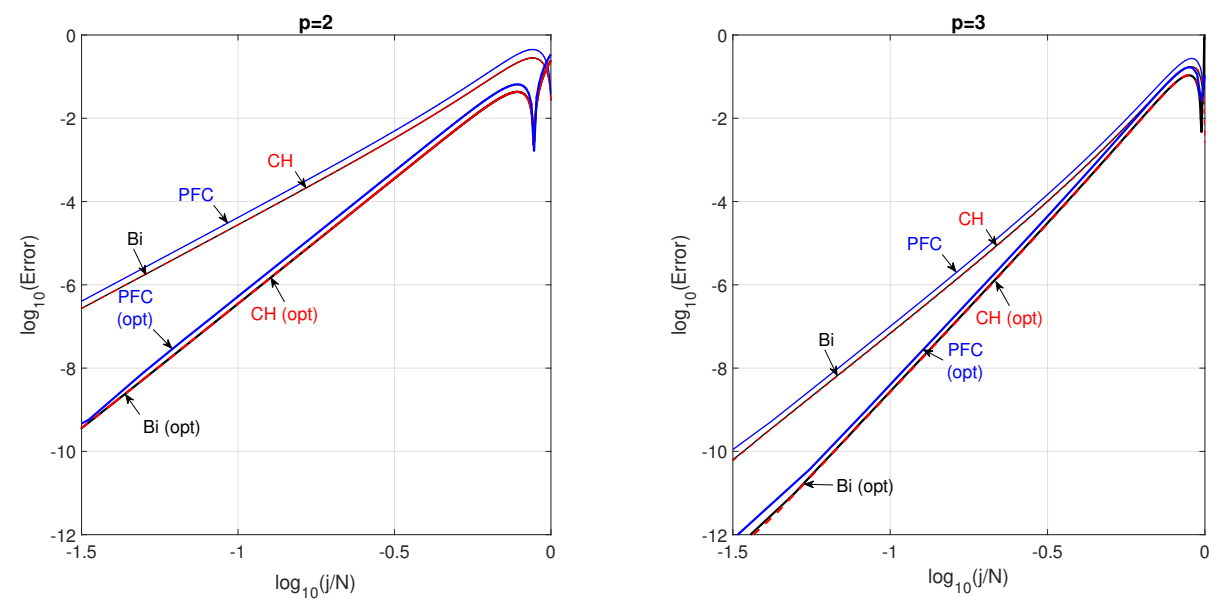

Figure 4: Relative approximation errors for the 1D biharmonic (Bi) equation with Dirichlet boundary conditions and Cahn-Hilliard $(\mathrm{CH})$ and phase-field crystal (PFC) equations with periodic boundary conditions using standard Gauss and optimal quadrature rules.

mixed isogeometric analysis with standard Gauss quadratures and optimally-blended rules for $2 \mathrm{D}$ linear, quadratic, and cubic elements. In these tables, we denote by $\mathrm{G}$ when using the standard Gauss quadrature rule while by $\mathrm{O}$ when the optimally-blended rule is applied. There are different optimally-blended rules and they lead to the same numerical results; see 1, 2, Herein, we use the $G_{p+1}-L_{p+1}$ optimally-blended rules. We also denote the convergence rates as $\rho_{p}$ for $p$-th order elements. For $p$-th order elements, we obtain 

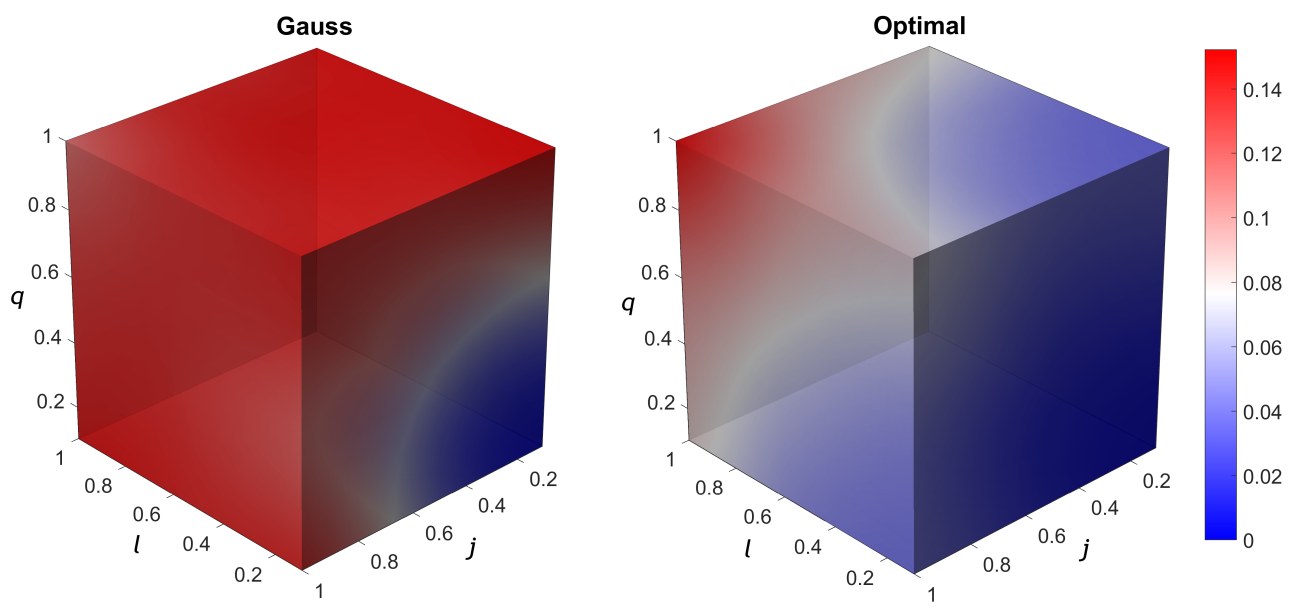

Figure 5: Relative approximation errors for quadratic $C^{1}$ elements for the biharmonic equations in 3D using mixed isogeometric analysis with Gauss quadratures (left) and optimally-blended rules (right).

convergence rates of order $2 p+2$ when using the optimally-blended rules. In the last columns of these tables, the rates 5.83 for $p=2$ and 7.18 for $p=3$ were calculated using $N=4,8,16,32$. We obtain $2 p+2$ when using $N=8,16,32$. The optimal rules deliver accurate approximations on coarse meshes while still in the pre-asymptotic range. These numerical experiments verify our theoretical findings.

Figure 6 shows the convergence of the eigenvalues $\lambda_{j}, j=1,2,4,8$ of the $2 \mathrm{D}$ biharmonic problem. The squared eigenvalue errors of the quadratic mixed isogeometric analysis with standard Gauss quadratures have convergence order close to 4 (that is $2 p$ ). Using optimally-blended rules leads to smaller eigenvalue errors and convergence order of 6 (that is $2 p+2$ ). Figure 7 shows the convergence of 2D Cahn-Hilliard operator eigenvalues when periodic boundary conditions are employed. Similar results are obtained for the phase-field equations.

\section{Conclusions and future outlook}

We present and study a mixed formulation of isogeometric analysis for a set of $2 n$-order differential eigenvalue problems, which includes operators arising from the biharmonic problem, Cahn-Hilliard, Swift-Hohenberg, and phase-field crystal equations. We show that the mixed formulation with standard quadrature rules applied for integrating the inner-products leads to optimal rates $(2 p)$ of convergence on eigenvalue errors while the mixed formulation with optimally-blended rules leads to super-convergent rates $2 p+2$ approximated eigenvalues. This work generalizes the theoretical results obtained for Laplacian eigenvalue problem to higher-order differential eigenvalue problems.

Future work in this direction includes generalization to other higher-order differential eigenvalue problem as well as to nonlinear differential eigenvalue problems. We expect the isogeometric discretization of nonlinear differential eigenvalue problems leads to nonlinear matrix eigenvalue problem, which requires advanced numerical solvers. Developing both optimally-blended quadrature rules for nonlinear differential eigenvalue problems and 


\begin{tabular}{|c|c|cc|cc|cc|cc|}
\hline$p$ & $N$ & \multicolumn{2}{|c|}{$e_{\lambda_{1}}$} & \multicolumn{2}{c|}{$e_{\lambda_{2}}$} & \multicolumn{2}{c|}{$e_{\lambda_{4}}$} & \multicolumn{2}{c|}{$e_{\lambda_{8}}$} \\
& & $\mathrm{G}$ & $\mathrm{O}$ & $\mathrm{G}$ & $\mathrm{O}$ & $\mathrm{G}$ & $\mathrm{O}$ & $\mathrm{G}$ & $\mathrm{O}$ \\
\hline \multirow{5}{*}{1} & 4 & $1.08 \mathrm{e}-1$ & $3.24 \mathrm{e}-3$ & $4.00 \mathrm{e}-1$ & $4.39 \mathrm{e}-2$ & $4.78 \mathrm{e}-1$ & $5.39 \mathrm{e}-2$ & $8.55 \mathrm{e}-1$ & $2.00 \mathrm{e}-1$ \\
& 8 & $2.60 \mathrm{e}-2$ & $1.99 \mathrm{e}-4$ & $9.10 \mathrm{e}-2$ & $2.63 \mathrm{e}-3$ & $1.08 \mathrm{e}-1$ & $3.24 \mathrm{e}-3$ & $2.08 \mathrm{e}-1$ & $1.26 \mathrm{e}-2$ \\
& 16 & $6.44 \mathrm{e}-3$ & $1.24 \mathrm{e}-5$ & $2.21 \mathrm{e}-2$ & $1.62 \mathrm{e}-4$ & $2.60 \mathrm{e}-2$ & $1.99 \mathrm{e}-4$ & $4.90 \mathrm{e}-2$ & $7.65 \mathrm{e}-4$ \\
& 32 & $1.61 \mathrm{e}-3$ & $7.74 \mathrm{e}-7$ & $5.48 \mathrm{e}-3$ & $1.01 \mathrm{e}-5$ & $6.44 \mathrm{e}-3$ & $1.24 \mathrm{e}-5$ & $1.21 \mathrm{e}-2$ & $4.74 \mathrm{e}-5$ \\
& $\rho_{1}$ & 2.02 & 4.01 & 2.06 & 4.03 & 2.07 & 4.03 & 2.05 & 4.02 \\
\hline \multirow{5}{*}{2} & 4 & $1.20 \mathrm{e}-3$ & $8.68 \mathrm{e}-5$ & $2.15 \mathrm{e}-2$ & $4.45 \mathrm{e}-3$ & $2.66 \mathrm{e}-2$ & $5.54 \mathrm{e}-3$ & $1.34 \mathrm{e}-1$ & $3.00 \mathrm{e}-2$ \\
& 8 & $6.83 \mathrm{e}-5$ & $1.34 \mathrm{e}-6$ & $9.74 \mathrm{e}-4$ & $6.97 \mathrm{e}-5$ & $1.20 \mathrm{e}-3$ & $8.68 \mathrm{e}-5$ & $5.23 \mathrm{e}-3$ & $7.18 \mathrm{e}-4$ \\
& 16 & $4.16 \mathrm{e}-6$ & $2.09 \mathrm{e}-8$ & $5.54 \mathrm{e}-5$ & $1.08 \mathrm{e}-6$ & $6.83 \mathrm{e}-5$ & $1.34 \mathrm{e}-6$ & $2.70 \mathrm{e}-4$ & $1.10 \mathrm{e}-5$ \\
& 32 & $2.59 \mathrm{e}-7$ & $3.26 \mathrm{e}-10$ & $3.38 \mathrm{e}-6$ & $1.68 \mathrm{e}-8$ & $4.16 \mathrm{e}-6$ & $2.09 \mathrm{e}-8$ & $1.60 \mathrm{e}-5$ & $1.71 \mathrm{e}-7$ \\
& $\rho_{2}$ & 4.06 & 6.01 & 4.20 & 6.01 & 4.21 & 6.01 & 4.34 & 5.83 \\
\hline \multirow{4}{*}{3} & 4 & $1.94 \mathrm{e}-5$ & $3.44 \mathrm{e}-6$ & $1.59 \mathrm{e}-3$ & $7.19 \mathrm{e}-4$ & $1.98 \mathrm{e}-3$ & $8.98 \mathrm{e}-4$ & $1.03 \mathrm{e}-2$ & $2.73 \mathrm{e}-3$ \\
& 8 & $2.60 \mathrm{e}-7$ & $1.47 \mathrm{e}-8$ & $1.61 \mathrm{e}-5$ & $3.13 \mathrm{e}-6$ & $2.01 \mathrm{e}-5$ & $3.91 \mathrm{e}-6$ & $2.14 \mathrm{e}-4$ & $7.46 \mathrm{e}-5$ \\
& 16 & $3.86 \mathrm{e}-9$ & $5.89 \mathrm{e}-11$ & $2.09 \mathrm{e}-7$ & $1.22 \mathrm{e}-8$ & $2.61 \mathrm{e}-7$ & $1.53 \mathrm{e}-8$ & $2.32 \mathrm{e}-6$ & $2.82 \mathrm{e}-7$ \\
& 32 & $5.95 \mathrm{e}-11$ & $2.23 \mathrm{e}-13$ & $3.10 \mathrm{e}-9$ & $4.81 \mathrm{e}-11$ & $3.86 \mathrm{e}-9$ & $6.00 \mathrm{e}-11$ & $3.24 \mathrm{e}-8$ & $1.09 \mathrm{e}-9$ \\
& $\rho_{3}$ & 6.10 & 7.96 & 6.32 & 7.95 & 6.32 & 7.95 & 6.13 & 7.18 \\
\hline
\end{tabular}

Table 2: Relative eigenvalue errors of mixed isogeometric analysis using standard Gauss (G) quadratures and optimally-blended $(\mathrm{O})$ rules for biharmonic eigenvalue problem.

\begin{tabular}{|c|c|cc|cc|cc|cc|}
\hline$p$ & $N$ & \multicolumn{2}{c}{$e_{\lambda_{1}}$} & \multicolumn{2}{c|}{$e_{\lambda_{2}}$} & \multicolumn{2}{c|}{$e_{\lambda_{4}}$} & \multicolumn{2}{c|}{$e_{\lambda_{8}}$} \\
& & $\mathrm{G}$ & $\mathrm{O}$ & $\mathrm{G}$ & $\mathrm{O}$ & $\mathrm{G}$ & $\mathrm{O}$ & $\mathrm{G}$ & $\mathrm{O}$ \\
\hline \multirow{5}{*}{1} & 4 & $1.05 \mathrm{e}-1$ & $3.16 \mathrm{e}-3$ & $3.96 \mathrm{e}-1$ & $4.34 \mathrm{e}-2$ & $4.75 \mathrm{e}-1$ & $5.36 \mathrm{e}-2$ & $8.51 \mathrm{e}-1$ & $1.99 \mathrm{e}-1$ \\
& 8 & $2.54 \mathrm{e}-2$ & $1.95 \mathrm{e}-4$ & $9.00 \mathrm{e}-2$ & $2.61 \mathrm{e}-3$ & $1.07 \mathrm{e}-1$ & $3.22 \mathrm{e}-3$ & $2.07 \mathrm{e}-1$ & $1.26 \mathrm{e}-2$ \\
& 16 & $6.29 \mathrm{e}-3$ & $1.21 \mathrm{e}-5$ & $2.19 \mathrm{e}-2$ & $1.60 \mathrm{e}-4$ & $2.58 \mathrm{e}-2$ & $1.98 \mathrm{e}-4$ & $4.88 \mathrm{e}-2$ & $7.62 \mathrm{e}-4$ \\
& 32 & $1.57 \mathrm{e}-3$ & $7.56 \mathrm{e}-7$ & $5.42 \mathrm{e}-3$ & $9.98 \mathrm{e}-6$ & $6.40 \mathrm{e}-3$ & $1.23 \mathrm{e}-5$ & $1.20 \mathrm{e}-2$ & $4.72 \mathrm{e}-5$ \\
& $\rho_{1}$ & 2.02 & 4.01 & 2.06 & 4.03 & 2.07 & 4.03 & 2.05 & 4.02 \\
\hline \multirow{5}{*}{2} & 4 & $1.17 \mathrm{e}-3$ & $8.47 \mathrm{e}-5$ & $2.13 \mathrm{e}-2$ & $4.40 \mathrm{e}-3$ & $2.64 \mathrm{e}-2$ & $5.51 \mathrm{e}-3$ & $1.34 \mathrm{e}-1$ & $2.99 \mathrm{e}-2$ \\
& 8 & $6.66 \mathrm{e}-5$ & $1.31 \mathrm{e}-6$ & $9.64 \mathrm{e}-4$ & $6.90 \mathrm{e}-5$ & $1.19 \mathrm{e}-3$ & $8.62 \mathrm{e}-5$ & $5.21 \mathrm{e}-3$ & $7.15 \mathrm{e}-4$ \\
& 16 & $4.06 \mathrm{e}-6$ & $2.04 \mathrm{e}-8$ & $5.49 \mathrm{e}-5$ & $1.07 \mathrm{e}-6$ & $6.78 \mathrm{e}-5$ & $1.33 \mathrm{e}-6$ & $2.69 \mathrm{e}-4$ & $1.10 \mathrm{e}-5$ \\
& 32 & $2.52 \mathrm{e}-7$ & $3.18 \mathrm{e}-10$ & $3.35 \mathrm{e}-6$ & $1.66 \mathrm{e}-8$ & $4.14 \mathrm{e}-6$ & $2.07 \mathrm{e}-8$ & $1.60 \mathrm{e}-5$ & $1.71 \mathrm{e}-7$ \\
& $\rho_{2}$ & 4.06 & 6.01 & 4.20 & 6.01 & 4.21 & 6.01 & 4.34 & 5.83 \\
\hline \multirow{4}{*}{3} & 4 & $1.90 \mathrm{e}-5$ & $3.35 \mathrm{e}-6$ & $1.57 \mathrm{e}-3$ & $7.12 \mathrm{e}-4$ & $1.97 \mathrm{e}-3$ & $8.92 \mathrm{e}-4$ & $1.02 \mathrm{e}-2$ & $2.72 \mathrm{e}-3$ \\
& 8 & $2.54 \mathrm{e}-7$ & $1.43 \mathrm{e}-8$ & $1.60 \mathrm{e}-5$ & $3.10 \mathrm{e}-6$ & $2.00 \mathrm{e}-5$ & $3.89 \mathrm{e}-6$ & $2.13 \mathrm{e}-4$ & $7.43 \mathrm{e}-5$ \\
& 16 & $3.77 \mathrm{e}-9$ & $5.75 \mathrm{e}-11$ & $2.07 \mathrm{e}-7$ & $1.21 \mathrm{e}-8$ & $2.59 \mathrm{e}-7$ & $1.52 \mathrm{e}-8$ & $2.31 \mathrm{e}-6$ & $2.80 \mathrm{e}-7$ \\
& 32 & $5.81 \mathrm{e}-11$ & $2.02 \mathrm{e}-13$ & $3.07 \mathrm{e}-9$ & $4.76 \mathrm{e}-11$ & $3.84 \mathrm{e}-9$ & $5.96 \mathrm{e}-11$ & $3.22 \mathrm{e}-8$ & $1.09 \mathrm{e}-9$ \\
& $\rho_{3}$ & 6.10 & 7.99 & 6.32 & 7.95 & 6.32 & 7.95 & 6.13 & 7.18 \\
\hline
\end{tabular}

Table 3: Relative eigenvalue errors of mixed isogeometric analysis using standard Gauss (G) quadratures and optimally-blended (O) rules for Cahn-Hilliard eigenvalue problem.

fast and efficient numerical solvers for their corresponding nonlinear matrix eigenvalue problems are subject to future work. 


\begin{tabular}{|c|c|cc|cc|ccc|cc|}
\hline$p$ & $N$ & \multicolumn{2}{|c|}{$e_{\lambda_{1}}$} & \multicolumn{2}{c|}{$e_{\lambda_{2}}$} & \multicolumn{2}{c|}{$e_{\lambda_{4}}$} & \multicolumn{2}{c|}{$e_{\lambda_{8}}$} \\
& & $\mathrm{G}$ & $\mathrm{O}$ & $\mathrm{G}$ & $\mathrm{O}$ & $\mathrm{G}$ & $\mathrm{O}$ & $\mathrm{G}$ & $\mathrm{O}$ \\
\hline \multirow{5}{*}{1} & 4 & $1.13 \mathrm{e}-1$ & $3.42 \mathrm{e}-3$ & $4.09 \mathrm{e}-1$ & $4.48 \mathrm{e}-2$ & $4.85 \mathrm{e}-1$ & $5.46 \mathrm{e}-2$ & $8.63 \mathrm{e}-1$ & $2.02 \mathrm{e}-1$ \\
& 8 & $2.74 \mathrm{e}-2$ & $2.10 \mathrm{e}-4$ & $9.29 \mathrm{e}-2$ & $2.69 \mathrm{e}-3$ & $1.09 \mathrm{e}-1$ & $3.28 \mathrm{e}-3$ & $2.10 \mathrm{e}-1$ & $1.27 \mathrm{e}-2$ \\
& 16 & $6.79 \mathrm{e}-3$ & $1.31 \mathrm{e}-5$ & $2.25 \mathrm{e}-2$ & $1.65 \mathrm{e}-4$ & $2.63 \mathrm{e}-2$ & $2.02 \mathrm{e}-4$ & $4.94 \mathrm{e}-2$ & $7.71 \mathrm{e}-4$ \\
& 32 & $1.69 \mathrm{e}-3$ & $8.16 \mathrm{e}-7$ & $5.59 \mathrm{e}-3$ & $1.03 \mathrm{e}-5$ & $6.53 \mathrm{e}-3$ & $1.26 \mathrm{e}-5$ & $1.21 \mathrm{e}-2$ & $4.77 \mathrm{e}-5$ \\
& $\rho_{1}$ & 2.02 & 4.01 & 2.06 & 4.03 & 2.07 & 4.03 & 2.05 & 4.02 \\
\hline \multirow{5}{*}{2} & 4 & $1.26 \mathrm{e}-3$ & $9.14 \mathrm{e}-5$ & $2.19 \mathrm{e}-2$ & $4.54 \mathrm{e}-3$ & $2.69 \mathrm{e}-2$ & $5.61 \mathrm{e}-3$ & $1.35 \mathrm{e}-1$ & $3.02 \mathrm{e}-2$ \\
& 8 & $7.19 \mathrm{e}-5$ & $1.41 \mathrm{e}-6$ & $9.94 \mathrm{e}-4$ & $7.11 \mathrm{e}-5$ & $1.22 \mathrm{e}-3$ & $8.79 \mathrm{e}-5$ & $5.27 \mathrm{e}-3$ & $7.24 \mathrm{e}-4$ \\
& 16 & $4.39 \mathrm{e}-6$ & $2.20 \mathrm{e}-8$ & $5.66 \mathrm{e}-5$ & $1.10 \mathrm{e}-6$ & $6.91 \mathrm{e}-5$ & $1.36 \mathrm{e}-6$ & $2.72 \mathrm{e}-4$ & $1.11 \mathrm{e}-5$ \\
& 32 & $2.72 \mathrm{e}-7$ & $3.43 \mathrm{e}-10$ & $3.45 \mathrm{e}-6$ & $1.71 \mathrm{e}-8$ & $4.22 \mathrm{e}-6$ & $2.11 \mathrm{e}-8$ & $1.61 \mathrm{e}-5$ & $1.73 \mathrm{e}-7$ \\
& $\rho_{2}$ & 4.06 & 6.01 & 4.20 & 6.01 & 4.21 & 6.01 & 4.34 & 5.83 \\
\hline \multirow{4}{*}{3} & 4 & $2.05 \mathrm{e}-5$ & $3.62 \mathrm{e}-6$ & $1.62 \mathrm{e}-3$ & $7.34 \mathrm{e}-4$ & $2.01 \mathrm{e}-3$ & $9.09 \mathrm{e}-4$ & $1.03 \mathrm{e}-2$ & $2.76 \mathrm{e}-3$ \\
& 8 & $2.74 \mathrm{e}-7$ & $1.54 \mathrm{e}-8$ & $1.65 \mathrm{e}-5$ & $3.20 \mathrm{e}-6$ & $2.04 \mathrm{e}-5$ & $3.96 \mathrm{e}-6$ & $2.15 \mathrm{e}-4$ & $7.52 \mathrm{e}-5$ \\
& 16 & $4.07 \mathrm{e}-9$ & $6.21 \mathrm{e}-11$ & $2.14 \mathrm{e}-7$ & $1.25 \mathrm{e}-8$ & $2.64 \mathrm{e}-7$ & $1.55 \mathrm{e}-8$ & $2.34 \mathrm{e}-6$ & $2.84 \mathrm{e}-7$ \\
& 32 & $6.26 \mathrm{e}-11$ & $1.76 \mathrm{e}-13$ & $3.17 \mathrm{e}-9$ & $4.90 \mathrm{e}-11$ & $3.91 \mathrm{e}-9$ & $6.08 \mathrm{e}-11$ & $3.26 \mathrm{e}-8$ & $1.10 \mathrm{e}-9$ \\
& $\rho_{3}$ & 6.10 & 8.08 & 6.32 & 7.95 & 6.32 & 7.95 & 6.13 & 7.18 \\
\hline
\end{tabular}

Table 4: Relative eigenvalue errors of mixed isogeometric analysis using standard Gauss (G) quadratures and optimally-blended (O) rules for Swift-Hohenberg eigenvalue problem.

\begin{tabular}{|c|c|cc|cc|cc|cc|}
\hline$p$ & $N$ & \multicolumn{2}{|c|}{$e_{\lambda_{1}}$} & \multicolumn{2}{c|}{$e_{\lambda_{2}}$} & \multicolumn{2}{c|}{$e_{\lambda_{4}}$} & \multicolumn{2}{c|}{$e_{\lambda_{8}}$} \\
& & $\mathrm{G}$ & $\mathrm{O}$ & $\mathrm{G}$ & $\mathrm{O}$ & $\mathrm{G}$ & $\mathrm{O}$ & $\mathrm{G}$ & $\mathrm{O}$ \\
\hline \multirow{5}{*}{1} & 4 & $1.08 \mathrm{e}-1$ & $3.24 \mathrm{e}-3$ & $4.00 \mathrm{e}-1$ & $4.39 \mathrm{e}-2$ & $4.78 \mathrm{e}-1$ & $5.39 \mathrm{e}-2$ & $8.55 \mathrm{e}-1$ & $2.00 \mathrm{e}-1$ \\
& 4 & $1.72 \mathrm{e}-1$ & $5.03 \mathrm{e}-3$ & $6.67 \mathrm{e}-1$ & $6.59 \mathrm{e}-2$ & $8.06 \mathrm{e}-1$ & $8.04 \mathrm{e}-2$ & $1.54 \mathrm{e} 0$ & $2.86 \mathrm{e}-1$ \\
& 8 & $4.07 \mathrm{e}-2$ & $3.10 \mathrm{e}-4$ & $1.42 \mathrm{e}-1$ & $4.00 \mathrm{e}-3$ & $1.67 \mathrm{e}-1$ & $4.90 \mathrm{e}-3$ & $3.30 \mathrm{e}-1$ & $1.90 \mathrm{e}-2$ \\
& 16 & $1.00 \mathrm{e}-2$ & $1.93 \mathrm{e}-5$ & $3.38 \mathrm{e}-2$ & $2.46 \mathrm{e}-4$ & $3.96 \mathrm{e}-2$ & $3.02 \mathrm{e}-4$ & $7.48 \mathrm{e}-2$ & $1.15 \mathrm{e}-3$ \\
& 32 & $2.50 \mathrm{e}-3$ & $1.20 \mathrm{e}-6$ & $8.34 \mathrm{e}-3$ & $1.53 \mathrm{e}-5$ & $9.76 \mathrm{e}-3$ & $1.88 \mathrm{e}-5$ & $1.82 \mathrm{e}-2$ & $7.14 \mathrm{e}-5$ \\
& $\rho_{1}$ & 2.03 & 4.01 & 2.10 & 4.02 & 2.12 & 4.02 & 2.13 & 3.99 \\
\hline \multirow{5}{*}{2} & 4 & $1.86 \mathrm{e}-3$ & $1.35 \mathrm{e}-4$ & $3.29 \mathrm{e}-2$ & $6.77 \mathrm{e}-3$ & $4.05 \mathrm{e}-2$ & $8.39 \mathrm{e}-3$ & $2.09 \mathrm{e}-1$ & $4.55 \mathrm{e}-2$ \\
& 8 & $1.06 \mathrm{e}-4$ & $2.08 \mathrm{e}-6$ & $1.48 \mathrm{e}-3$ & $1.06 \mathrm{e}-4$ & $1.82 \mathrm{e}-3$ & $1.31 \mathrm{e}-4$ & $7.90 \mathrm{e}-3$ & $1.08 \mathrm{e}-3$ \\
& 16 & $6.47 \mathrm{e}-6$ & $3.24 \mathrm{e}-8$ & $8.43 \mathrm{e}-5$ & $1.64 \mathrm{e}-6$ & $1.03 \mathrm{e}-4$ & $2.03 \mathrm{e}-6$ & $4.07 \mathrm{e}-4$ & $1.66 \mathrm{e}-5$ \\
& 32 & $4.02 \mathrm{e}-7$ & $5.06 \mathrm{e}-10$ & $5.14 \mathrm{e}-6$ & $2.55 \mathrm{e}-8$ & $6.30 \mathrm{e}-6$ & $3.16 \mathrm{e}-8$ & $2.42 \mathrm{e}-5$ & $2.58 \mathrm{e}-7$ \\
& $\rho_{2}$ & 4.06 & 6.01 & 4.21 & 6.01 & 4.21 & 6.01 & 4.35 & 5.83 \\
\hline \multirow{5}{*}{3} & 4 & $3.02 \mathrm{e}-5$ & $5.34 \mathrm{e}-6$ & $2.42 \mathrm{e}-3$ & $1.09 \mathrm{e}-3$ & $3.00 \mathrm{e}-3$ & $1.36 \mathrm{e}-3$ & $1.55 \mathrm{e}-2$ & $4.12 \mathrm{e}-3$ \\
& 8 & $4.04 \mathrm{e}-7$ & $2.28 \mathrm{e}-8$ & $2.45 \mathrm{e}-5$ & $4.76 \mathrm{e}-6$ & $3.04 \mathrm{e}-5$ & $5.92 \mathrm{e}-6$ & $3.22 \mathrm{e}-4$ & $1.12 \mathrm{e}-4$ \\
& 16 & $6.00 \mathrm{e}-9$ & $9.12 \mathrm{e}-11$ & $3.18 \mathrm{e}-7$ & $1.86 \mathrm{e}-8$ & $3.94 \mathrm{e}-7$ & $2.31 \mathrm{e}-8$ & $3.50 \mathrm{e}-6$ & $4.24 \mathrm{e}-7$ \\
& 32 & $9.09 \mathrm{e}-11$ & $9.10 \mathrm{e}-13$ & $4.71 \mathrm{e}-9$ & $7.23 \mathrm{e}-11$ & $5.84 \mathrm{e}-9$ & $9.09 \mathrm{e}-11$ & $4.88 \mathrm{e}-8$ & $1.66 \mathrm{e}-9$ \\
& $\rho_{3}$ & 6.11 & 7.54 & 6.32 & 7.95 & 6.32 & 7.95 & 6.14 & 7.17 \\
\hline
\end{tabular}

Table 5: Relative eigenvalue errors of mixed isogeometric analysis using standard Gauss (G) quadratures and optimally-blended $(\mathrm{O})$ rules for phase-field crystal eigenvalue problem.

\section{${ }_{241}$ Acknowledgement}

242 This publication was made possible in part by the CSIRO Professorial Chair in Com243 putational Geoscience at Curtin Universitylænd the Deep Earth Imaging Enterprise Fu- 

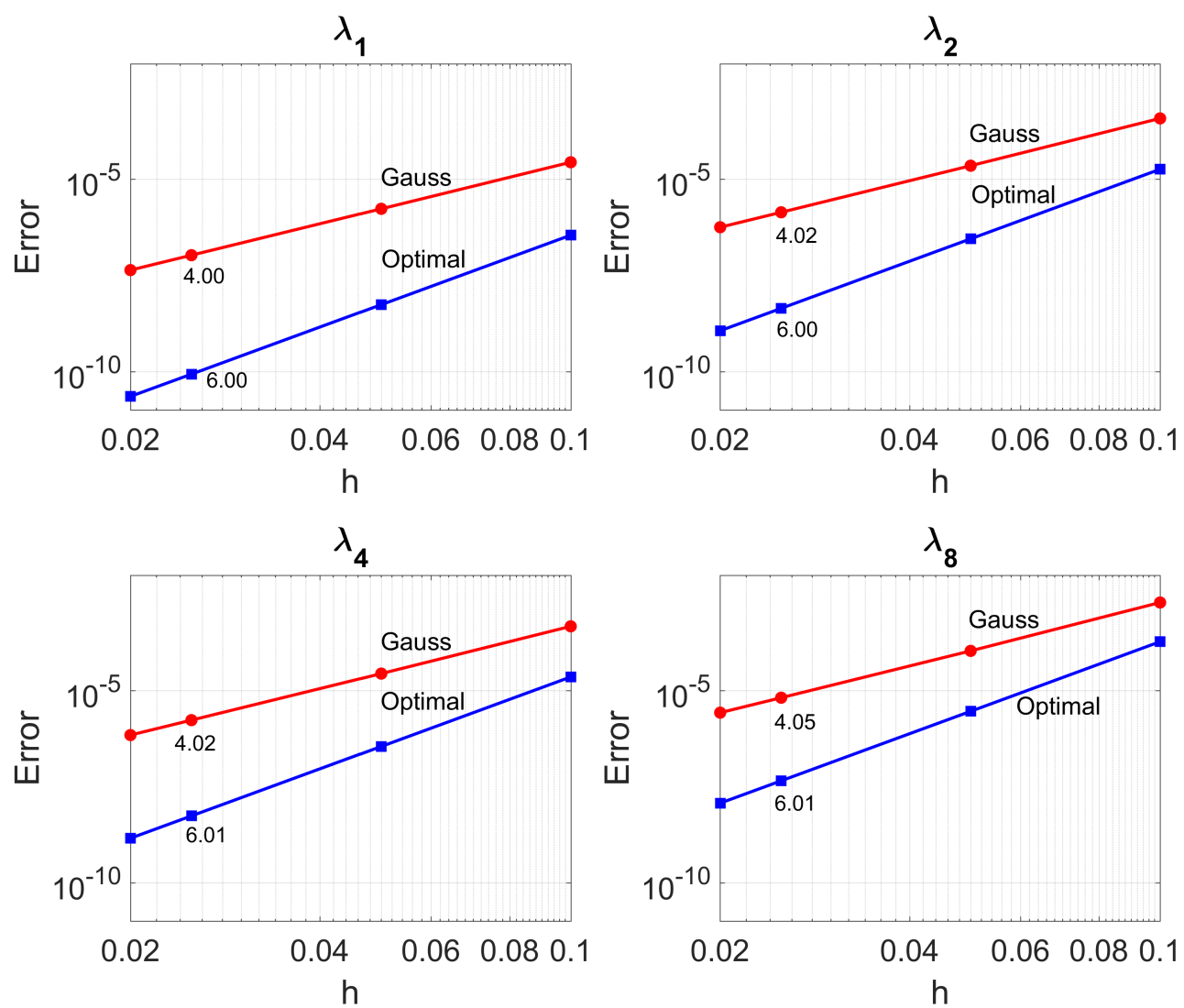

Figure 6: The convergence of the eigenvalues $\lambda_{j}, j=1,2,4,8$ of the 2D biharmonic problem (simply supported plate).

ture Science Platforms of the Commonwealth Scientific Industrial Research Organisation, CSIRO, of Australia. Additional support was provided by the European Union's Horizon 2020 Research and Innovation Program of the Marie Skłodowska-Curie grant agreement No. 777778, the Mega-grant of the Russian Federation Government (N 14.Y26.31.0013), the Institute for Geoscience Research (TIGeR), and the Curtin Institute for Computation. The J. Tinsley Oden Faculty Fellowship Research Program at the Institute for Computational Engineering and Sciences (ICES) of the University of Texas at Austin has partially supported the visits of VMC to ICES.

\section{References}

[1] V. Puzyrev, Q. Deng, V. M. Calo, Dispersion-optimized quadrature rules for isogeometric analysis: modified inner products, their dispersion properties, and optimally blended schemes, Computer Methods in Applied Mechanics and Engineering 320 (2017) 421-443.

[2] V. M. Calo, Q. Deng, V. Puzyrev, Dispersion optimized quadratures for isogeometric analysis, arXiv preprint arXiv:1702.04540. 

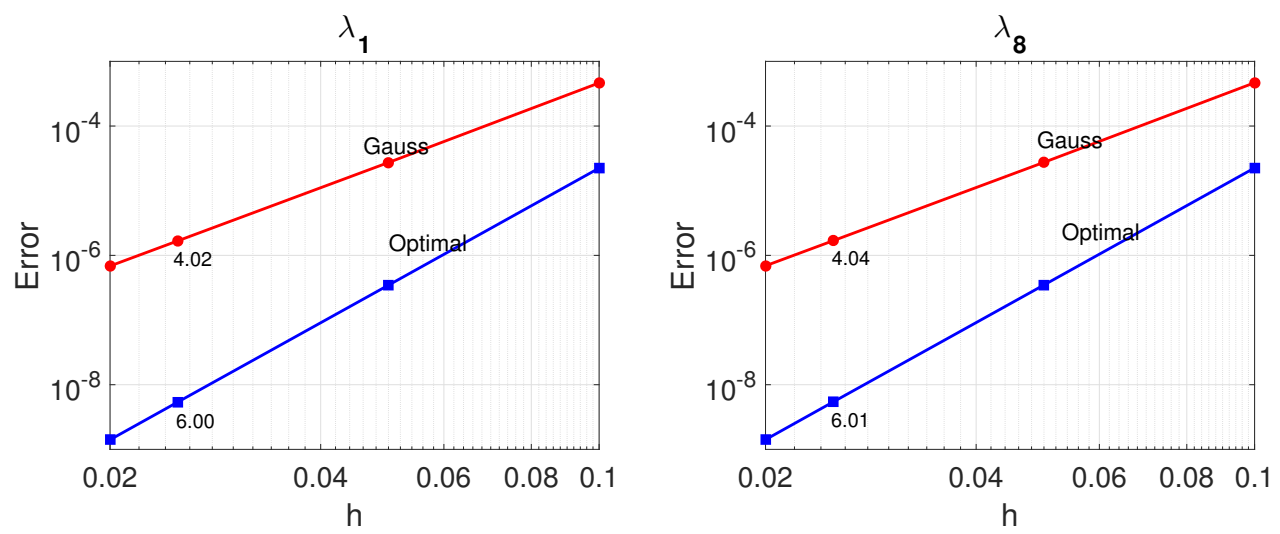

Figure 7: The convergence of the eigenvalues $\lambda_{1}$ and $\lambda_{8}$ of the 2D Cahn-Hilliard equation with periodic boundary conditions.

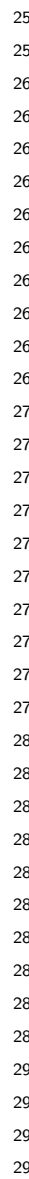

[3] F. Brezzi, On the existence, uniqueness and approximation of saddle-point problems arising from Lagrangian multipliers, Revue française d'automatique, informatique, recherche opérationnelle. Analyse numérique 8 (R2) (1974) 129-151.

[4] D. S. Malkus, T. J. R. Hughes, Mixed finite element methods - reduced and selective integration techniques: a unification of concepts, Computer Methods in Applied Mechanics and Engineering 15 (1) (1978) 63-81.

[5] F. Brezzi, M. Fortin, Mixed and hybrid finite element methods, Springer Science \& Business Media, 1991.

[6] F. Auricchio, F. Brezzi, C. Lovadina, Mixed finite element methods, Encyclopedia of computational mechanics.

[7] G. N. Gatica, A simple introduction to the mixed finite element method, Theory and Applications. Springer Briefs in Mathematics. Springer, London.

[8] I. Babuška, The finite element method with Lagrangian multipliers, Numerische Mathematik 20 (3) (1973) 179-192.

[9] M. Crouzeix, P.-A. Raviart, Conforming and nonconforming finite element methods for solving the stationary Stokes equations, Revue française d'automatique informatique recherche opérationnelle. Mathématique 7 (R3) (1973) 33-75.

[10] M. Cervera, M. Chiumenti, R. Codina, Mixed stabilized finite element methods in nonlinear solid mechanics: Part II: Strain localization, Computer Methods in Applied Mechanics and Engineering 199 (37) (2010) 2571-2589.

[11] J. Wang, X. Ye, A weak Galerkin mixed finite element method for second order elliptic problems, Mathematics of Computation 83 (289) (2014) 2101-2126.

[12] E. T. Chung, Y. Efendiev, C. S. Lee, Mixed generalized multiscale finite element methods and applications, Multiscale Modeling \& Simulation 13 (1) (2015) 338-366.

[13] V. John, A. Linke, C. Merdon, M. Neilan, L. G. Rebholz, On the divergence constraint in mixed finite element methods for incompressible flows, SIAM Review 59 (3) (2017) 492-544.

[14] L. P. Franca, T. J. R. Hughes, Two classes of mixed finite element methods, Computer Methods in Applied Mechanics and Engineering 69 (1) (1988) 89-129.

[15] J. Douglas, J. P. Wang, An absolutely stabilized finite element method for the Stokes problem, Mathematics of computation 52 (186) (1989) 495-508.

[16] N. Kechkar, D. Silvester, Analysis of locally stabilized mixed finite element methods for the Stokes problem, Mathematics of Computation 58 (197) (1992) 1-10.

[17] A. Masud, T. J. R. Hughes, A stabilized mixed finite element method for Darcy flow, Computer methods in applied mechanics and engineering 191 (39) (2002) 4341-4370.

[18] C. R. Dohrmann, P. B. Bochev, A stabilized finite element method for the Stokes problem based on polynomial pressure projections, International Journal for Numerical Methods in Fluids 46 (2) 
(2004) 183-201.

[19] V. M. Calo, A. Romkes, E. Valseth, Automatic variationally stable analysis for FE computations: An introduction, arXiv preprint arXiv:1808.01888.

[20] R. S. Falk, J. E. Osborn, Error estimates for mixed methods, RAIRO. Analyse numérique 14 (3) (1980) 249-277.

[21] D. N. Arnold, F. Brezzi, Mixed and nonconforming finite element methods: implementation, postprocessing and error estimates, ESAIM: Mathematical Modelling and Numerical Analysis 19 (1) (1985) 7-32.

[22] J. Donea, S. Giuliani, J.-P. Halleux, An arbitrary Lagrangian-Eulerian finite element method for transient dynamic fluid-structure interactions, Computer methods in applied mechanics and engineering 33 (1-3) (1982) 689-723.

[23] T. J. R. Hughes, G. N. Wells, Conservation properties for the Galerkin and stabilised forms of the advection-diffusion and incompressible Navier-Stokes equations, Computer Methods in Applied Mechanics and Engineering 194 (9-11) (2005) 1141-1159.

[24] A. Buffa, C. De Falco, G. Sangalli, Isogeometric analysis: stable elements for the 2D Stokes equation, International Journal for Numerical Methods in Fluids 65 (11-12) (2011) 1407-1422.

[25] J. A. Evans, T. J. R. Hughes, Isogeometric divergence-conforming B-splines for the steady NavierStokes equations, Mathematical Models and Methods in Applied Sciences 23 (08) (2013) 1421-1478.

[26] T. Hoang, C. V. Verhoosel, F. Auricchio, E. H. van Brummelen, A. Reali, Mixed isogeometric finite cell methods for the Stokes problem, Computer Methods in Applied Mechanics and Engineering 316 (2017) 400-423.

[27] A. F. Sarmiento, A. M. Côrtes, D. Garcia, L. Dalcin, N. Collier, V. M. Calo, PetIGA-MF: a multifield high-performance toolbox for structure-preserving B-splines spaces, Journal of Computational Science 18 (2017) 117-131.

[28] R. Dudu, L. L. Lavier, T. J. R. Hughes, V. M. Calo, A finite Eulerian formulation for compressible and nearly incompressible hyperelasticity using high-order B-spline finite elements, International Journal for Numerical Methods in Engineering 89 (6) (2012) 762-785.

[29] B. Dortdivanlioglu, A. Krischok, L. Beirão da Veiga, C. Linder, Mixed isogeometric analysis of strongly coupled diffusion in porous materials, International Journal for Numerical Methods in Engineering 114 (1) (2018) 28-46.

[30] Y. W. Bekele, E. Fonn, T. Kvamsdal, A. M. Kvarving, S. Nordal, On mixed isogeometric analysis of poroelasticity, arXiv preprint arXiv:1706.01275.

[31] T. Elguedj, Y. Bazilevs, V. M. Calo, T. J. R. Hughes, B and F and projection methods for nearly incompressible linear and non-linear elasticity and plasticity using higher-order nurbs elements, Computer methods in applied mechanics and engineering 197 (33) (2008) 2732-2762.

[32] L. Espath, A. Sarmiento, P. Vignal, B. Varga, A. Cortes, L. Dalcin, V. M. Calo, Energy exchange analysis in droplet dynamics via the navier-stokes-cahn-hilliard model, Journal of Fluid Mechanics 797 (2016) 389-430.

[33] P. M. Bleher, J. L. Lebowitz, E. R. Speer, Existence and positivity of solutions of a fourth-order nonlinear PDE describing interface fluctuations, Communications on Pure and Applied Mathematics 47 (7) (1994) 923-942.

[34] M. D. Korzec, P. L. Evans, A. Münch, B. Wagner, Stationary solutions of driven fourth-and sixthorder Cahn-Hilliard-type equations, SIAM Journal on Applied Mathematics 69 (2) (2008) 348-374.

[35] C. V. Verhoosel, M. A. Scott, T. J. Hughes, R. De Borst, An isogeometric analysis approach to gradient damage models, International Journal for Numerical Methods in Engineering 86 (1) (2011) $115-134$.

[36] P. Vignal, L. Dalcin, D. L. Brown, N. Collier, V. M. Calo, An energy-stable convex splitting for the phase-field crystal equation, Computers \& Structures 158 (2015) 355-368.

[37] P. Vignal, N. Collier, L. Dalcin, D. L. Brown, V. M. Calo, An energy-stable time-integrator for phase-field models, Computer Methods in Applied Mechanics and Engineering 316 (2017) 11791214.

[38] P. Torres, Z. Cheng, J. Ren, Non-degeneracy and uniqueness of periodic solutions for 2n-order differential equations, Discrete Contin. Dyn. Syst., Ser. A 33 (2013) 2155-2168.

[39] M. N. Guddati, B. Yue, Modified integration rules for reducing dispersion error in finite element methods, Computer methods in applied mechanics and engineering 193 (3) (2004) 275-287.

[40] M. Ainsworth, H. A. Wajid, Optimally blended spectral-finite element scheme for wave propagation and nonstandard reduced integration, SIAM Journal on Numerical Analysis 48 (1) (2010) 346-371.

[41] Q. Deng, M. Bartoň, V. Puzyrev, V. M. Calo, Dispersion-minimizing quadrature rules for C1 quadratic isogeometric analysis, Computer Methods in Applied Mechanics and Engineering 328 
(2018) 554-564.

[42] S. M. Allen, J. W. Cahn, A microscopic theory for antiphase boundary motion and its application to antiphase domain coarsening, Acta Metallurgica 27 (6) (1979) 1085-1095.

[43] J. W. Cahn, J. E. Hilliard, Free energy of a nonuniform system. I. Interfacial free energy, The Journal of chemical physics 28 (2) (1958) 258-267.

[44] J. Swift, P. C. Hohenberg, Hydrodynamic fluctuations at the convective instability, Physical Review A 15 (1) (1977) 319.

[45] T. V. Savina, A. A. Golovin, S. H. Davis, A. A. Nepomnyashchy, P. W. Voorhees, Faceting of a growing crystal surface by surface diffusion, Physical Review E 67 (2) (2003) 021606.

[46] K. R. Elder, M. Katakowski, M. Haataja, M. Grant, Modeling elasticity in crystal growth, Physical review letters 88 (24) (2002) 245701.

[47] P. G. Ciarlet, Finite Element Method for Elliptic Problems, Society for Industrial and Applied Mathematics, Philadelphia, PA, USA, 2002.

[48] S. P. Timoshenko, S. Woinowsky-Krieger, Theory of plates and shells, McGraw-hill, 1959.

[49] A. Andreev, R. Lazarov, M. Racheva, Postprocessing and higher order convergence of the mixed finite element approximations of biharmonic eigenvalue problems, Journal of computational and applied mathematics 182 (2) (2005) 333-349.

[50] I. Babuška, J. Osborn, Eigenvalue problems, in: Handbook of Numerical Analysis, Vol. II, Handb. Numer. Anal., II, North-Holland, Amsterdam, 1991, pp. 641-787.

[51] E. B. Davies, L $^{p}$ spectral theory of higher-order elliptic differential operators, Bulletin of the London Mathematical Society 29 (5) (1997) 513-546.

[52] K. Ishihara, A mixed finite element method for the biharmonic eigenvalue problems of plate bending, Publications of the Research Institute for Mathematical Sciences 14 (2) (1978) 399-414.

[53] R. A. Adams, Sobolev spaces, Academic Press, New York, 1975.

[54] C. De Boor, A practical guide to splines, Vol. 27, Springer-Verlag New York, 1978.

[55] L. Piegl, W. Tiller, The NURBS book, Springer Science \& Business Media, 1997.

[56] M. Barton̆, V. M. Calo, Optimal quadrature rules for odd-degree spline spaces and their application to tensor-product-based isogeometric analysis, Computer Methods in Applied Mechanics and Engineering 305 (2016) 217-240.

[57] M. Bartoň, V. M. Calo, Gaussian quadrature for splines via homotopy continuation: rules for C2 cubic splines, Journal of Computational and Applied Mathematics 296 (2016) 709-723.

[58] M. Bartoň, V. M. Calo, Gauss-Galerkin quadrature rules for quadratic and cubic spline spaces and their application to isogeometric analysis, Computer-Aided Design 82 (2017) 57-67.

[59] V. M. Calo, M. Cicuttin, Q. Deng, A. Ern, Spectral approximation of elliptic operators by the hybrid high-order method, arXiv preprint arXiv:1711.01135.

[60] M. Bartoň, V. M. Calo, Q. Deng, V. Puzyrev, Generalization of the Pythagorean Eigenvalue Error Theorem and its Application to Isogeometric Analysis, SEMA-SIMAI Springer Series, 2018.

[61] Q. Deng, V. Calo, Dispersion-minimized mass for isogeometric analysis, Computer Methods in Applied Mechanics and Engineering 341 (2018) 71-92.

[62] F. Odeh, J. B. Keller, Partial differential equations with periodic coefficients and bloch waves in crystals, Journal of Mathematical Physics 5 (11) (1964) 1499-1504.

[63] J. A. Cottrell, A. Reali, Y. Bazilevs, T. J. R. Hughes, Isogeometric analysis of structural vibrations, Computer methods in applied mechanics and engineering 195 (41) (2006) 5257-5296.

[64] V. Puzyrev, Q. Deng, V. M. Calo, Spectral approximation properties of isogeometric analysis with variable continuity, Computer Methods in Applied Mechanics and Engineering 334 (2018) 22-39. 